\title{
Review
}

\section{Macroalgal Defense against Competitors and Herbivores}

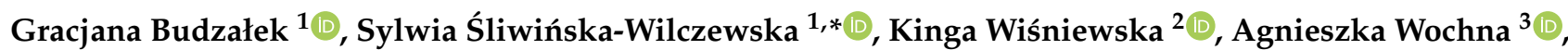 \\ Iwona Bubak $^{4}\left(\mathbb{D}\right.$, Adam Latała ${ }^{1}$ and Józef Maria Wiktor ${ }^{5}(\mathbb{D}$
}

1 Division of Marine Ecosystems Functioning, Institute of Oceanography, University of Gdańsk, P-81-378 Gdynia, Poland; gbudzalek@gmail.com (G.B.); oceal@ug.edu.pl (A.L.)

2 Division of Marine Chemistry and Environmental Protection, Institute of Oceanography, University of Gdańsk, P-81-378 Gdynia, Poland; kinga.wisniewska@phdstud.ug.edu.pl

3 GIS Centre, Institute of Oceanography, University of Gdańsk, P-81-378 Gdynia, Poland; agnieszka.wochna@ug.edu.pl

4 Division of Hydrology, Institute of Geography, University of Gdansk, P-80-309 Gdańsk, Poland; iwona.bubak@ug.edu.pl

5 Department of Marine Ecology, Institute of Oceanology of the Polish Academy of Sciences, P-81-779 Sopot, Poland; wiktor@iopan.gda.pl

* Correspondence: ocessl@ug.edu.pl; Tel.: +48-58-5236894

Citation: Budzałek, G.;

Śliwińska-Wilczewska, S.;

Wiśniewska, K.; Wochna, A.; Bubak,

I.; Latała, A.; Wiktor, J.M. Macroalgal Defense against Competitors and Herbivores. Int. J. Mol. Sci. 2021, 22, 7865. https://doi.org/10.3390/ ijms22157865

Academic Editor: Zhixiang Chen

Received: 17 June 2021

Accepted: 22 July 2021

Published: 23 July 2021

Publisher's Note: MDPI stays neutral with regard to jurisdictional claims in published maps and institutional affiliations.

Copyright: (c) 2021 by the authors. Licensee MDPI, Basel, Switzerland. This article is an open access article distributed under the terms and conditions of the Creative Commons Attribution (CC BY) license (https:// creativecommons.org/licenses/by/ $4.0 /)$.
Abstract: Macroalgae are the source of many harmful allelopathic compounds, which are synthesized as a defense strategy against competitors and herbivores. Therefore, it can be predicted that certain species reduce aquaculture performance. Herein, the allelopathic ability of 123 different taxa of green, red, and brown algae have been summarized based on literature reports. Research on macroalgae and their allelopathic effects on other animal organisms was conducted primarily in Australia, Mexico, and the United States. Nevertheless, there are also several scientific reports in this field from South America and Asia; the study areas in the latter continents coincide with areas where aquaculture is highly developed and widely practiced. Therefore, the allelopathic activity of macroalgae on coexisting animals is an issue that is worth careful investigation. In this work, we characterize the distribution of allelopathic macroalgae and compare them with aquaculture locations, describe the methods for the study of macroalgal allelopathy, present the taxonomic position of allelopathic macroalgae and their impact on coexisting aquatic competitors (Cnidaria) and herbivores (Annelida, Echinodermata, Arthropoda, Mollusca, and Chordata), and compile information on allelopathic compounds produced by different macroalgae species. This work gathers the current knowledge on the phenomenon of macroalgal allelopathy and their allelochemicals affecting aquatic animal (competitors and predators) worldwide and it provides future research directions for this topic.

Keywords: aquatic animals; allelopathy; allelochemicals; chemical defense; defense strategy; plant defense; species interactions

\section{Introduction}

Aquaculture has rapidly grown over the past few decades and is now the fastestgrowing food sector worldwide [1]. The global aquaculture production in 2015 was approximately 106 million tons, which represents approximately 163 billion US dollars [2]. The global population has been increasing and is expected to reach $\sim 10$ billion in the middle of the 21st century [3]. The corresponding increase in food demand is driving the expansion of aquaculture [4]. The pressure on these food sectors to maximize production and reduce losses is also expected to increase [2].

A popular method to increase aquaculture production is to enrich farming tanks with macroalgae species. Macroalgae as a food source believed to be an ideal candidate for growth in fishponds because they provide high biomass production and protein content [5]. Additionally, the environment of the ponds is improved by macroalgae through the balance 
of $\mathrm{pH}$ levels [6]. Different macroalgal species have been integrated into land-based integrated multi-trophic aquacultures (IMTA) for biomass production [7]. The high amount of protein from macroalgae represents valuable feed for animal species with high commercial value $[5,7]$. However, studies on this topic rarely mention that allelopathic macroalgae can negatively affect and even exterminate both competitors and predators by secreting a broad range of harmful and toxic substances such as acetogenins, alkaloids, aromatic compounds, fluorotannins, polyphenols, terpenes, and amino acids [8].

Macroalgal allelopathy refers to the effects of substances produced by the microalgae on target organisms [9]. These effects can be related to the growth, health, origin, or population biology of the donor and target organisms $[8,9]$. The allelopathic activity of macroalgae is a complex process. It is considered that its level depends on the production of active allelopathic compounds and their effective escalation to accompanying organisms [10]. Macroalgae are mainly benthic organisms firmly attached to the seabed, which forces them to compete for substrates, nutrients, and light with other benthic organisms. There are also unattached forms of macroalgae [11], which can influence the development of planktonic organisms. Kersen [11] showed that the unattached forms of Furcellaria lumbricalis and Coccotylus truncatus can be considerably denser than their respective attached forms. Therefore, their deleterious effects on other organisms can be stronger than those of benthic algae. Nevertheless, their allelopathic activities have not been sufficiently investigated.

Studies related to the impact of macroalgae on other organisms have mainly focused on marine environments $[8,12,13]$. However, freshwater and brackish macroalgae can also achieve rapid biomass increase, which can result in algal blooms [14-16]. Moreover, macroalgae from freshwater and brackish ecosystems can negatively affect the growth of photoautotrophs $[17,18]$. Nevertheless, there is little research on the impact of these organisms on coexisting aquatic animals. Macroalgae in marine environments belong to three groups: Ulvophyceae, Chlorophyta (green algae), Florideophyceae, Rhodophyta (red algae), and Phaeophyceae, Ochrophyta (formerly Phaeophyta; brown algae), whereas those from freshwater include mainly Ulvophyceae, Chlorophyta and Charophyceae, Charophyta [19]. Macroalgae with confirmed allelopathic activity against other heterotrophic organisms are shown in Figure 1.
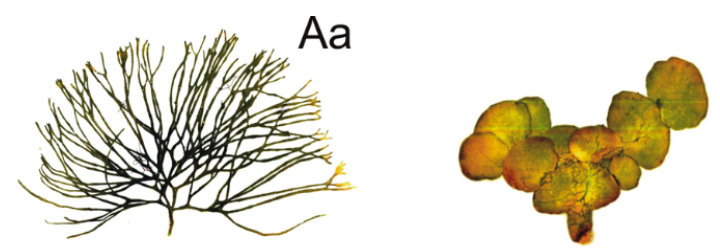

$A b$
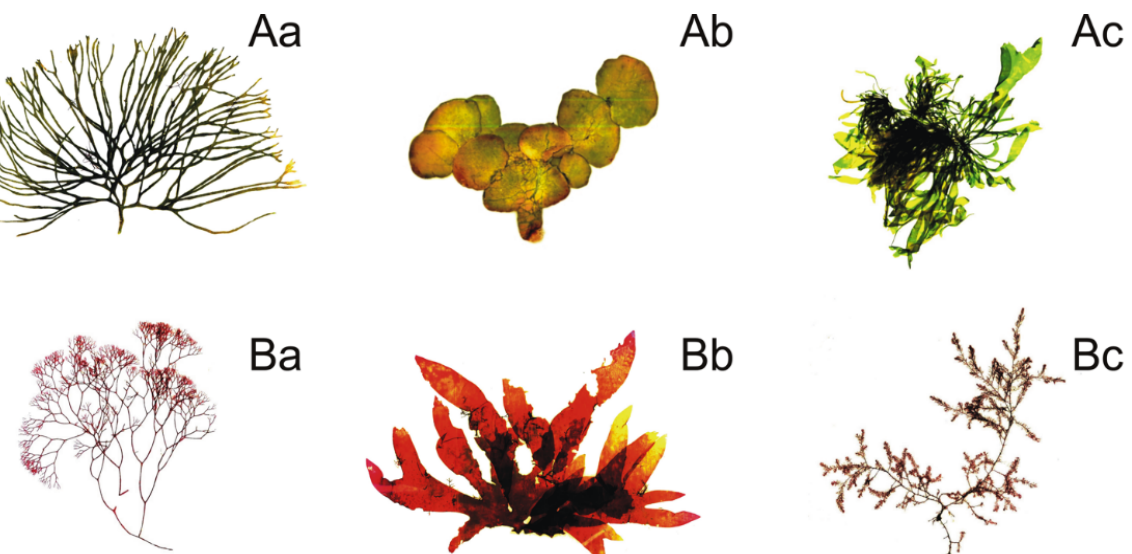

$\mathrm{Ba}$
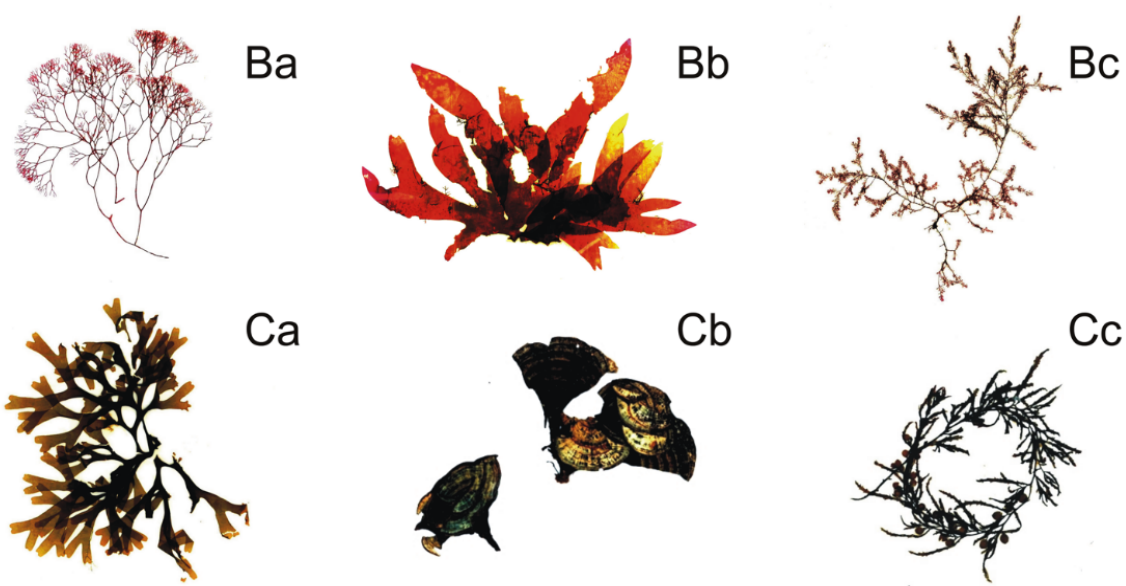

Figure 1. Examples of allelopathic green algae (A): Codium fragile (a), Halimeda tuna (b), Ulva sp. (c); red algae. (B): Ceramium rubrum (a), Grateloupia sp. (b), Polysiphonia sp. (c); brown algae. (C): Dictyota sp. (a), Padina sp. (b), Sargassum sp. (c). 
Recently, research on the allelopathy phenomenon has increased significantly $[8,13,20]$; however, to the best of our knowledge, no published review has revealed the negative effects of macroalgae on coexisting competitors and predators. In this work, we (i) characterize the distribution of allelopathic macroalgae and compare them with aquaculture locations, (ii) describe the methods for the study of macroalgal allelopathy, (iii) present the taxonomic position of allelopathic macroalgae and their impact on coexisting animal competitors (Cnidaria species) and herbivores (Annelida, Echinodermata, Arthropoda, Mollusca, and Chordata species), and (iv) compile information on allelopathic compounds produced by different macroalgae species. This work gathers the current knowledge on the phenomenon of macroalgal allelochemicals affecting aquatic competitors and herbivores worldwide and it provides future research directions for this topic.

\section{Distribution of Allelopathic Macroalgae and Aquaculture Locations}

In this work, the allelopathic effect of green algae (Chlorophyta, Ulvophyceae), red algae (Rhodophyta, Florideophyceae), and brown algae (Ochrophyta, Phaeophyceae) was investigated against different aquatic animals. Allelopathic activity has been reported for a total of 123 taxa, including 37 green algae (30\%), 45 red algae (37\%), and 41 brown algae (33\%). The allelopathic ability of 11 different genera of Chlorophyta, 28 genera of Rhodophyta, and 13 genera of Ochrophyta has been reported (Figure S1, Table S1). The allelopathic activity of macroalgae has most often been studied in Chlorophyta from the genera Caulerpa, Chlorodesmis, and Ulva. Hypnea sp. has been the most frequently studied among Rhodophyta for allelopathy. Among the allelopathic Ochrophyta, Dictyota sp. and Lobophora sp. have been the most frequently studied. The least numerous studies for allelopathic ability have been conducted for organisms belonging to Anadyomene, Codium, Penicillus, and Rhiphilia (green algae); Asparagopsis, Callophycus, Centroceras, Ceramium, Chondria, Chondriopsis, Chondrophycus, Crassiphycus, Delisea, Dermonema, Digenea, Endosiphonia, Peyssonnelia, Phacelocarpus, Plocamium, Polysiphonia, Tayloriella, Tichocarpus, and Yuzurua (red algae); and Canistrocarpus, Desmarestia, Dictyopteris, Dilophus, Ecklonia, Laminaria, and Sphacelaria sp. (brown algae).

Research on macroalgae and their allelopathic effects on other organisms has been primarily conducted in Australia, Mexico, and the United States (Figure 2). Nevertheless, a few scientific investigations have been conducted in South America and Asia in areas coinciding with aquaculture activity (Figure 2). In most areas, all three phyla were tested. However, the studies in some regions focused only on one macroalgae phylum. Chlorodesmis fastigiata is the most studied green algae, accounting for $30.4 \%$ of all tested organisms of this phylum [21-26]. In studies on brown algae, Dictyota bartayresiana dominates, accounting for $12.5 \%$ of the total studies [22,24,27], whereas in red algae, Galaxaura filamentosa is the most widely investigated, with studies accounting for 13.6\% [22-24]. 


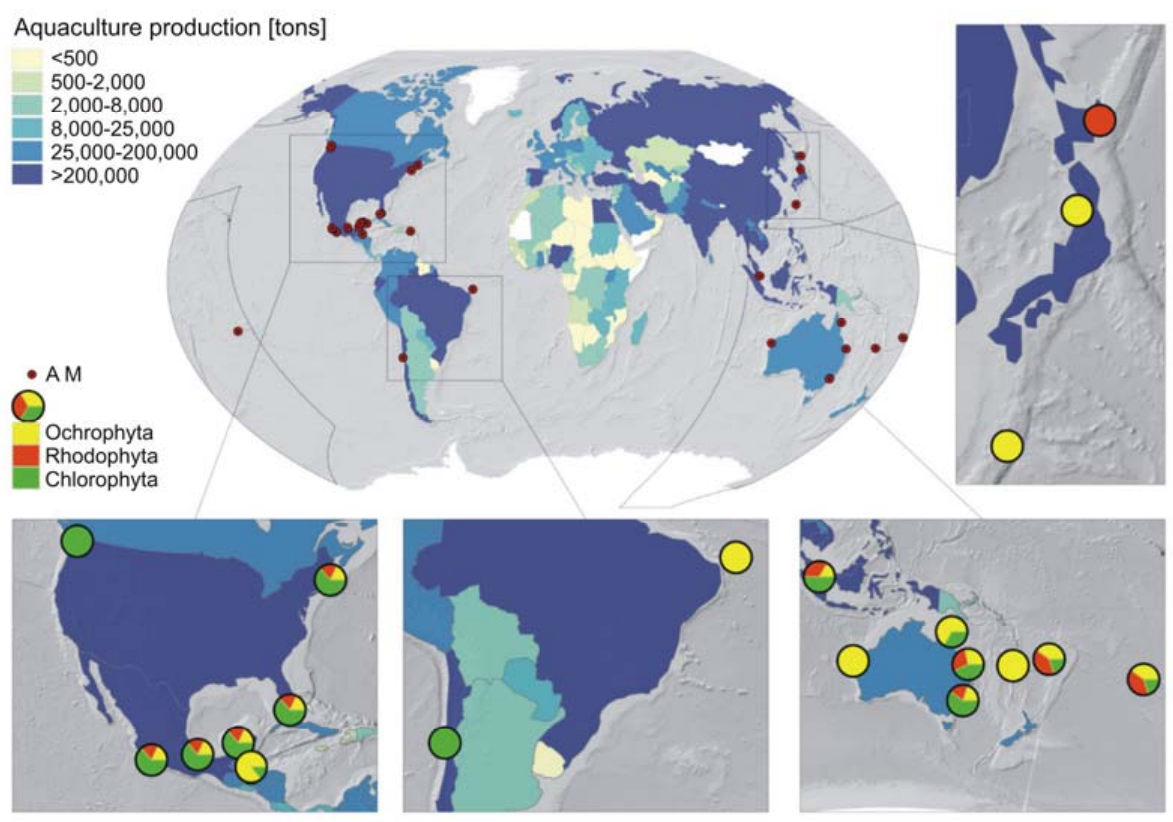

Figure 2. Allelopathic macroalgae (AM) in the studied areas based on the donor species found in the literature compared to the places where world aquaculture production occurs (based on the World Bank data; https:/ / data.worldbank.org/indicator/ER.FSH.AQUA.MT, accessed on 17 June 2021).

\section{Methods for Macroalgal Allelopathy Examination}

To recognize the allelopathy impact of macroalgae on coexisting aquatic animals (competitors and herbivores), many investigation methods are necessary, from field observation to co-culturing experiments in mesocosms. Most studies on the allelopathic activity of macroalgae on target aquatic animals are characterized by a specific method suited to test those organisms and environment. Four main methods for testing macroalgal allelopathy are shown in Figure 3. In the most used method, the recruitment plate method, the impact of macroalgae on animals is examined by observing the settlement degree of target organisms and their survival rate on specially arranged tiles placed in the field $[21,23,28,29]$. In the second most-used method, the effect of macroalgal extracts or exudates on the development and survival of target animals is analyzed [8,30-39]. The third method includes the analysis of the interaction of macroalgae or their compounds on animals tested in a petri dish $[40,41]$. Finally, experiments in mesocosms or arranged co-culturing experiments for algae and animals are conducted $[25,27,42]$.

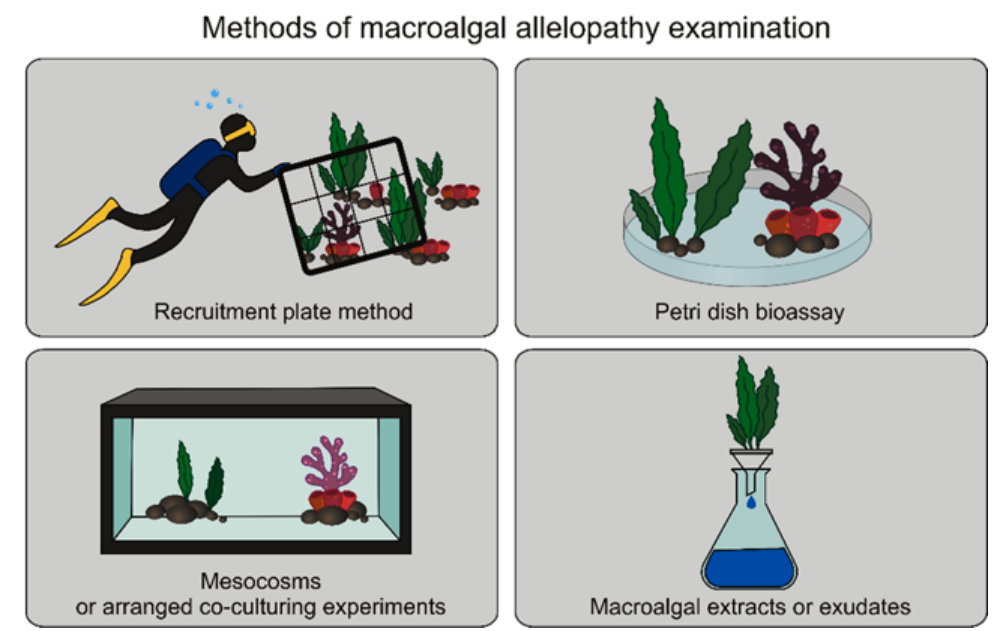

Figure 3. Most used methods to investigate the allelopathy phenomenon. 


\section{Taxonomic Position of Allelopathic Macroalgae and Their Impact on Coexisting Competitors and Herbivores}

Macroalgae are major competitors for the light and space for corals and other benthic organisms from the Cnidaria phylum on tropical reefs [43]. Competition can occur through direct and indirect physical and chemical mechanisms reviewed in detail by Chadwick and Morrow [44]. Macroalgae can produce inhibitory compounds affecting corals and epibionts that compete for light or space [9]. Globally, many coral reefs have been damaged, and areas with reduced coral cover and increased macroalgal abundance have been widely identified [45]. Despite the well-documented negative correlation between macroalgae and coral recruitment, the mechanisms through which macroalgae affect this recruitment have received little attention.

In addition, macroalgal allelopathy has an important and as-yet unrecognized role in structuring temperate shallow marine communities of herbivores: Annelida (e.g., Sabellaria cementarium and Spinoidae sp.) [41], Echinodermata (e.g., Holopneustes purpurascens, Lytechinus variegates, and Strongylocentrotus intermedius) $[31,33,35,36]$, and Arthropoda species (Cancer oregonensis, Metacarcinus magister, and Pachygrapsus transversus) $[35,46]$. Furthermore, several researchers have reported the negative effects of macroalgae on Mollusca species e.g., [38,47,48]; they suggested that green macroalgae species (especially from the Ulvophyceae class) can inhibit the growth and development of co-occurring organisms from the genus Crassostrea. Moreover, oyster larvae (e.g., Crassostrea gigas) are susceptible to extracts from Ulvaria lactuca thallus at relatively low concentrations [48]. Although several researchers have reported both negative and positive effects of green algae species on invertebrates $[41,46,49,50]$, few studies have reported the potential effects of Ulva sp. on the economically relevant Mollusca, Crassostrea virginica [38]. Many aquaculture farms cultivate $C$. virginica in areas where Ulva is present. Research has also shown that macroalgae can adversely affect species belonging to the Chordata phylum [8,30-32]. Moreover, certain investigated fishes that belong to Carassius sp. and Tilapia sp. are consumed by humans. As contribution of aquatic animals to global food is crucial, such results are alarming and warrant special attention [2].

The interactions of green algae on 13 different genera of aquatic animals (both competitors and predators) have also been reported (Figure 4). The allelopathic activity of Chlorophyta species was tested against six taxa belonging to Cnidaria, two to Mollusca, two to Annelida, two to Arthropoda, and one to Chordata phylum. Conversely, the influence of red algae was investigated on ten aquatic animals (five belonging to Cnidaria, two to Annellida, two to Echinodermata, and one to Chordata). Overall, the greatest number of animal species have been tested for their sensitivity to brown algae. The allelopathic activity of these macroalgae was tested against 19 genera of different aquatic animals. Allelopathic activity of brown algae was tested on animals belonging to the Cnidaria, Mollusca, Annelida, Echinodermata, Arthropoda, and Chordata phyla. As in the case of other macroalgae, the allelopathic activity of brown algae has been most frequently studied for taxa belonging to the Cnidaria. Animals belonging to the genus Crassostrea and Haliotis (Mollusca), Strongylocentrotus (Echinodermata), Cancer and Metacarcinus (Arthropoda) as well as Carassius and Tilapia (Chordata), are commonly used in aquaculture. Therefore, it is important to further investigate and compare information on the interactions between macroalgal species and economically important animals. 


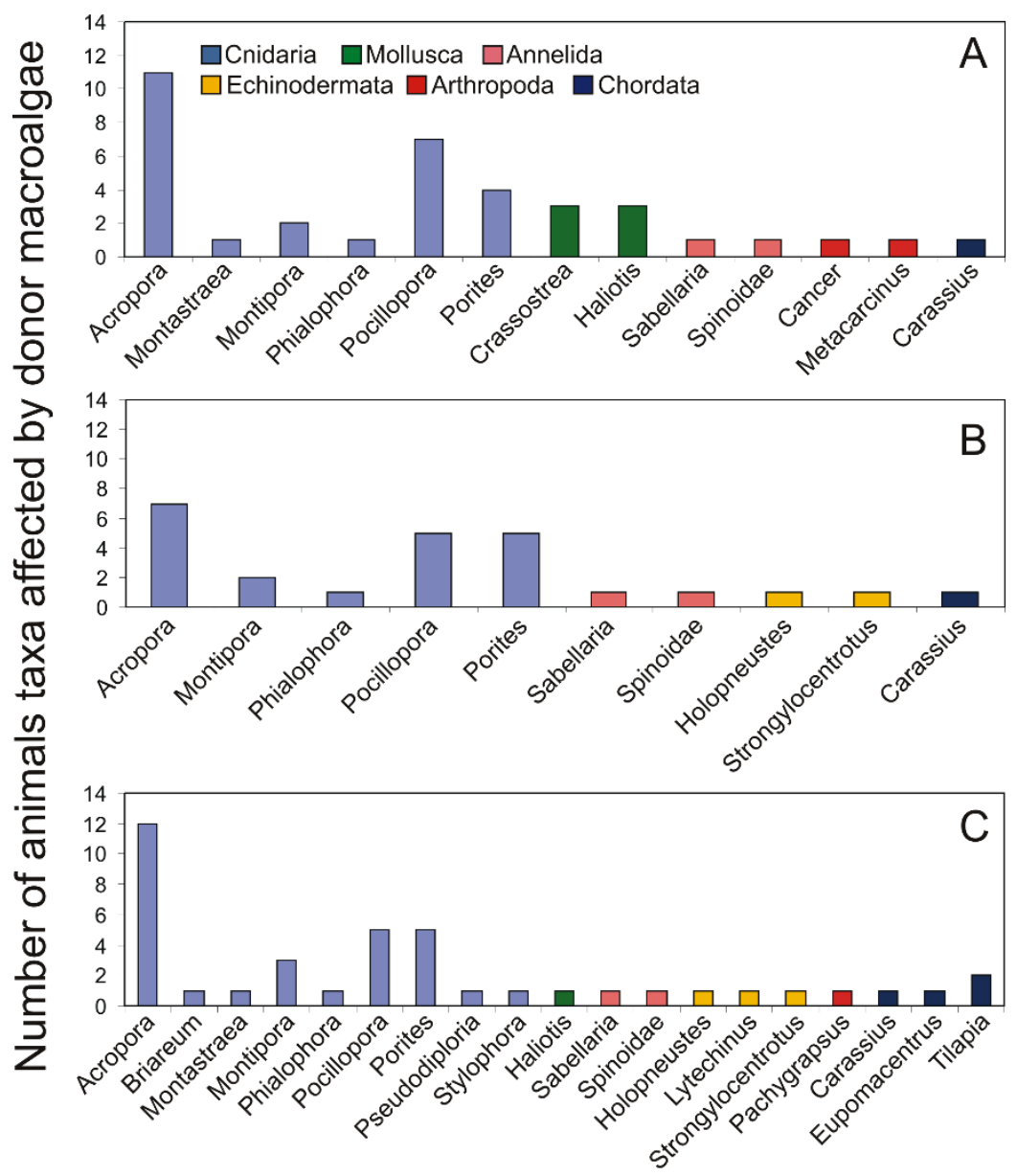

Figure 4. Number of target competitors and herbivores affected by green algae (A), red algae (B), and brown algae $(\mathbf{C})$, based on taxa found in the literature.

\subsection{The Allelopathic Activity of Green Algae}

The allelopathic activity of green algae (Ulvophyceae, Chlorophyta) was confirmed by several authors (Table 1). Studies have shown that the presence of green algae has a generally negative effect on Cnidaria [21-26,28,39,51,52]. Tanner [21] was the first author who showed that Chlorodesmis fastigiata and Halimeda sp. had a negative impact on Acropora (Isopora) cuneata, Acropora hrueggemanni, Acropora palifera, and Pocillopora damicornis. Similar research was conducted by Rasher et al. [22]. Andras et al. [51] proved that the green alga Rhiphilia pencilloides caused coral bleaching when placed in contact with Porites rus. Morrow et al. [52] showed the impact of macroalgal extracts obtained from Halimeda tuna on the sublethal stress response of corals. In turn, Bonaldo and Hay [23] investigated macroalgae-coral interactions considering both non-allelopathic and allelopathic species. Furthermore, Lee et al. [28] examined the effects of macroalgal species on the settlement success of $P$. damicomis larvae under aquarium conditions. Ritson-Williams et al. [24] examined that $C$. fastigiata negatively affects $A$. millepora, $M$. digitata, and $P$. damicornis. Fong et al. [39] showed that the mortality of Pocillopora acuta larvae increased significantly with an increase in the concentration of the crude extract obtained from Bryopsis sp. Longo and Hay [26] demonstrated that the lipid-soluble extracts obtained from the green alga $C$. fastigiata suppressed coral Pocillopora verrucosa photochemical efficiency. Conversely, Del Monaco et al. [25] showed that donor macroalgae $C$. fastigiata damages corals via allelopathy regardless of $\mathrm{CO}_{2}$ concentration. Only Birrell et al. [40] described a positive and neutral effect of Chlorophyta on Cnidaria. These authors demonstrated that $C$. fastigiata caused a slight delay in the settlement of coral larvae; however, these results were not statistically significant. Green-Gavrielidis et al. [38], Nelson et al. [47], and Nelson and Greg [48] have 
shown that macroalgae from the genus Ulva have had a negative impact on Mollusca. Green-Gavrielidis et al. [38] showed that bloom-forming Ulva compressa negatively affected the growth of Crassostrea virginica and the strongest effect was seen in larvae exposed to $U$. compressa exudates growing on nutrient-sufficient medium. Nelson et al. [47] and Nelson and Greg [48] showed that oyster larvae (Crassostrea gigas) are susceptible to extracts from dried Ulva lactuca and Ulvaria obscura at relatively low concentrations. Conversely, Muñoz et al. [50] showed that the presence of Ulva sp. improved the growth rate of the Haliotis rufescens larvae, while Huggett et al. [49] noted high colonization of Haliotis rubra in the presence of Ulva australis, Ulva compressa, and Ulvaria obscura. Warkus et al. [41] were the only authors who studied the influence of Ulvophyceae on Annelida (Table 1). This work demonstrated the negative effect of Chaetomorpha sp., Codium fragile, Ulva sp. (formerly Enteromorpha sp.), and Ulva lactuca on polychaeta Sabellaria cementarium and Spinoidae sp. In turn, the diverse effects of Ulvaria obscura on Arthropoda have been described by Van Alstyne et al. [46]. The authors demonstrated that tested green algae did not affect the survival of Cancer oregonensis and Metacarcinus magister juveniles. It was also shown that $U$. obscura had little effect on the time of first molting of these animals. Alvarez-Hernández et al. [8] showed that various species belonging to Chlorophyta were considered highly toxic to Chordata (the goldfish Carassius auratus auratus) when acetonic or ethanolic extract was made. The most toxic Chlorophyta were: Caulerpa cupressoides, Caulerpa racemosa, Chaetomorpha antennina, and Penicillus capitatus. However, aqueous extract obtained from these green algae had no effect on C. auratus auratus (Table 1 ).

Table 1. Examples of allelopathic activity of green algae against competitors and herbivores.

\begin{tabular}{|c|c|c|c|}
\hline Donor Chlorophyta & Target Organism-Cnidaria & Effect & References \\
\hline Bryopsis corymbose & Pocillopora damicornis & - & Lee et al. [28] \\
\hline Bryopsis sp. & Pocillopora acuta & - & Fong et al. [39] \\
\hline \multirow[t]{2}{*}{ Chlorodesmis fastigiata } & Acropora millepora & $+/ 0$ & Birrell et al. [40] \\
\hline & Acropora aspera & - & \multirow{4}{*}{ Bonaldo and Hay [23] } \\
\hline \multirow{3}{*}{ Chlorodesmis fastigiata } & Pocillopora damicornis & - & \\
\hline & Porites cylindrica & - & \\
\hline & Porites lobata & - & \\
\hline Chlorodesmis fastigiata & Acropora intermedia & - & Del Monaco et al. [25] \\
\hline \multirow[t]{2}{*}{ Chlorodesmis fastigiata } & Phialophora verrucosa & - & Longo and Hay [26] \\
\hline & Acropora millepora & - & \multirow{4}{*}{ Rasher et al. [22] } \\
\hline \multirow[t]{3}{*}{ Chlorodesmis fastigiata } & Montipora digitata & - & \\
\hline & Pocillopora damicornis & - & \\
\hline & Acropora millepora & - & \\
\hline \multirow[t]{3}{*}{ Chlorodesmis fastigiata } & Montipora digitata & - & \multirow[t]{3}{*}{ Ritson-Williams et al. [24] } \\
\hline & Pocillopora damicornis & - & \\
\hline & Acropora cuneata & - & \\
\hline \multirow{3}{*}{ Chlorodesmis fastigiata } & Acropora hrueggemanni & - & \multirow{3}{*}{ Tanner [21] } \\
\hline & Acropora pnlifera & - & \\
\hline & Pocillopora damicornis & - & \\
\hline Halimeda opuntia & Pocillopora damicornis & - & Lee et al. [28] \\
\hline \multirow{3}{*}{ Halimeda tuna } & Montastraea faveolate & - & \multirow{3}{*}{ Morrow et al. [52] } \\
\hline & Porites astreoides & 0 & \\
\hline & Acropora cuneata & - & \\
\hline \multirow{3}{*}{ Halimeda sp. } & Acropora hrueggemanni & - & \multirow{3}{*}{ Tanner [21] } \\
\hline & Acropora pnlifera & - & \\
\hline & Pocillopora damicornis & - & \\
\hline Rhiphilia pencilloides & Porites rus & - & Andras et al. [51] \\
\hline
\end{tabular}


Table 1. Cont.

\begin{tabular}{|c|c|c|c|}
\hline Donor Chlorophyta & Target Organism-Mollusca & Effect & References \\
\hline Ulva australis & Haliotis rubra & + & Huggett et al. [49] \\
\hline Ulva compressa & Crassostrea virginica & - & Green-Gavrielidis et al. [38] \\
\hline Ulva compressa & Haliotis rubra & + & Huggett et al. [49] \\
\hline Ulva fenestrata & Crassostrea gigas & - & Nelson et al. [47] \\
\hline Ulva lactuca & Crassostrea virginica & - & Green-Gavrielidis et al. [38] \\
\hline Ulvaria lactuca & Crassostrea gigas & - & Nelson and Gregg [48] \\
\hline Ulva lens & Crassostrea gigas & - & Nelson et al. [47] \\
\hline Ulvaria obscura & Haliotis rubra & + & Huggett et al. [49] \\
\hline Ulva obscura & Crassostrea virginica & - & Green-Gavrielidis et al. [38] \\
\hline Ulvaria obscura & Crassostrea gigas & - & Nelson and Gregg [48] \\
\hline Ulva sp. & Haliotis rufescens & + & Muñoz et al. [50] \\
\hline Donor Chlorophyta & Target Organism-Annelida & Effect & References \\
\hline Chaetomorpha sp. & $\begin{array}{l}\text { Sabellaria cementarium } \\
\text { Spinoidae sp. }\end{array}$ & $\begin{array}{l}- \\
-\end{array}$ & Warkus et al. [41] \\
\hline Codium fragile & $\begin{array}{l}\text { Sabellaria cementarium } \\
\text { Spinoidae sp. }\end{array}$ & $\begin{array}{l}- \\
-\end{array}$ & Warkus et al. [41] \\
\hline Ulva (Enteromorpha) sp. & $\begin{array}{l}\text { Sabellaria cementarium } \\
\text { Spinoidae sp. }\end{array}$ & $\begin{array}{l}- \\
-\end{array}$ & Warkus et al. [41] \\
\hline Ulva lactuca & $\begin{array}{l}\text { Sabellaria cementarium } \\
\text { Spinoidae sp. }\end{array}$ & - & Warkus et al. [41] \\
\hline Donor Chlorophyta & Target Organism-Arthropoda & Effect & References \\
\hline Ulvaria obscura & $\begin{array}{c}\text { Cancer oregonensis } \\
\text { Metacarcinus magister }\end{array}$ & $\begin{array}{l}0 /- \\
0 /-\end{array}$ & Van Alstyne et al. [46] \\
\hline Donor Chlorophyta & Target Organism-Chordata & Effect & References \\
\hline Anadyomene stellata & Carassius auratus auratus & $0 /-$ & Alvarez-Hernández et al. [8] \\
\hline Caulerpa cupressoides & Carassius auratus auratus & $0 /-$ & Alvarez-Hernández et al. [8] \\
\hline Caulerpa paspaloides & Carassius auratus auratus & $0 /-$ & Alvarez-Hernández et al. [8] \\
\hline Caulerpa racemosa & Carassius auratus auratus & $0 /-$ & Alvarez-Hernández et al. [8] \\
\hline Chaetomorpha antennina & Carassius auratus auratus & $0 /-$ & Alvarez-Hernández et al. [8] \\
\hline Penicillus capitatus & Carassius auratus auratus & $0 /-$ & Alvarez-Hernández et al. [8] \\
\hline
\end{tabular}

Note: - means inhibiting effects, + means stimulating effect, 0 - means lack of effect.

Many macroalgae, such as Ulva sp., are cosmopolitan organisms, and in nutrientrich coastal waters, they are often dominant and bloom-forming species $[15,53,54]$. These studies confirm that Chlorophyta may have a negative impact on co-occurring animal organisms. Therefore, allelopathy phenomenon of species belonging to Chlorophyta on coexisting animal organisms should be widely studied in the future.

\subsection{The Allelopathic Activity of Red Algae}

The allelopathic activity of red algae (Florideophyceae, Rhodophyta) on coexisting animals has also been confirmed by a few experimental studies (Table 2). The negative effect of red algae on Cnidaria was described by Tanner [21], Rasher et al. [22], Bonaldo and Hay [23], Ritson-Williams et al. [24], Del Monaco et al. [25], Longo and Hay [26], Fong et al. [39], and Andras et al. [51]. In addition, a few authors [22,24,39,42] observed that certain red algae species had no allelopathic effect on target Cnidaria (Table 2). Tanner [21] described that Acropora species growing faster in areas from which red macroalgae Peyssonnelia sp. had been removed compared to control areas where Rhodophyta species were present. Similarly, Andras et al. [51] used field experiments to show that contact with the red algae Callophycus densus, Phacelocarpus neurymenioides, and Plocamium pacificum induces bleaching on natural colonies of Porites rus. Moreover, the corals in the control experiments, in which they encountered plastic imitation algae, showed no bleaching, 
which may suggest the effect of the red macroalgae allelochemicals rather than the effect of shading or physical contact. Bonaldo and Hay, [23] demonstrated that the presence of allelopathic red macroalgae Galaxaura filamentosa caused faster and more extensive damage to Acropora aspera and P. damicornis than to Porites cylindrica, Porites lobata, and Montipora digitata. Furthermore, Longo and Hay [26] showed that the red algae Amansia rhodantha and Asparagopsis taxiformis extracts negatively affected the photochemical efficiency of the coral Phialophora verrucosa. Fong et al. [39] examined the effects of crude extracts from macroalgal species Endosiphonia horrida and Hypnea pannosa on Pocillopora acuta larvae. In turn, Del Monaco et al. [25] showed that common Rhodophyta Amansia glomerata damage corals Acropora intermedia via allelopathy, however, the effect of the macroalgal extracts was not stronger when the tested Rhodophyta species were grown under elevated $\mathrm{CO}_{2}$ conditions. Rasher et al. [22] and Ritson-Williams et al. [24] showed that red algae G. filamentosa had negative effects on Acropora millepora, M. digitate, and P. damicornis. Similarly, Kuffner et al. [42] demonstrated no allelopathic effects of Chondrophycus poiteaui (formerly Laurencia poiteaui) on the recruitment success of Porites astreoides larvae. Moreover, Warkus et al. [41] described the negative influence of Rhodophyta Grateloupia turu turu and Polysiphonia denudata on Annelida Sabellaria cementarium and Spinoidae sp. Ishii et al. [36] also demonstrated that compounds obtained from red algae (Tichocarpus crinitus) exhibited feeding-deterrent properties against the Echinodermata Strongylocentrotus intermedius. Conversely, Williamson et al. [33] showed that allelochemicals produced by Delisea pulchra caused a positive effect on metamorphosis and triggered settlement in other Echinodermeta Holopneustes purpurascens. The studies by Alvarez-Hernández et al. [8] showed that, in general, the aqueous extract did not affect the behavior of the Carassius auratus auratus belonging to Chordata phylum. The only exception was Chondriopsis dasyphylla f. pyrifera, which showed strong toxicity to the tested animal after exposure to aqueous, acetonic, and ethanolic extracts. The studies by Alvarez-Hernández et al. [8] showed that the activity of macroalgae also depends on the place of occurrence of individual species.

Table 2. Examples of allelopathic activity of red algae against competitors and herbivores.

\begin{tabular}{|c|c|c|c|}
\hline Donor Rhodophyta & Target Organism-Cnidaria & Effect & References \\
\hline \multirow{3}{*}{ Amphiroa crassa } & Acropora millepora & - & \multirow{3}{*}{$\begin{array}{l}\text { Rasher et al. [22], } \\
\text { Ritson-Williams et al. [24] }\end{array}$} \\
\hline & Montipora digitata & 0 & \\
\hline & Pocillopora damicornis & - & \\
\hline Amansia glomerata & Acropora intermedia & - & Del Monaco et al. [25] \\
\hline Amansia rhodantha & Phialophora verrucosa & - & Longo and Hay [26] \\
\hline Asparagopsis taxiformis & Phialophora verrucosa & - & Longo and Hay [26] \\
\hline Callophycus densus & Porites rus & - & Andras et al. [51] \\
\hline Chondrophycus poiteaui & Porites astreoides & 0 & Kuffner et al. [42] \\
\hline \multirow[t]{2}{*}{ Endosiphonia horrida } & Pocillopora acuta & - & Fong et al. [39] \\
\hline & Acropora millepora & - & \multirow{4}{*}{$\begin{array}{l}\text { Rasher et al. [22], } \\
\text { Ritson-Williams et al. [24] }\end{array}$} \\
\hline \multirow[t]{3}{*}{ Galaxaura filamentosa } & Montipora digitata & - & \\
\hline & Pocillopora damicornis & - & \\
\hline & Acropora aspera & - & \\
\hline \multirow{3}{*}{ Galaxaura filamentosa } & Pocillopora damicornis & - & \multirow{3}{*}{ Bonaldo and Hay [23] } \\
\hline & Porites cylindrica & - & \\
\hline & Porites lobata & - & \\
\hline \multirow[t]{2}{*}{ Hypnea pannosa } & Pocillopora acuta & 0 & Fong et al. [39] \\
\hline & Acropora millepora & - & \multirow{3}{*}{$\begin{array}{l}\text { Rasher et al. [22], } \\
\text { Ritson-Williams et al. [24] }\end{array}$} \\
\hline \multirow[t]{2}{*}{ Liagora sp. } & Montipora digitata & 0 & \\
\hline & Pocillopora damicornis & - & \\
\hline Phacelocarpus neurymenioides & Porites rus & - & \multirow{3}{*}{$\begin{array}{l}\text { Andras et al. [51] } \\
\text { Andras et al. [51] }\end{array}$} \\
\hline Plocamium pacificum & Porites rus & - & \\
\hline \multirow{4}{*}{ Peyssonnelia sp. } & Acropora cuneata & - & \\
\hline & Acropora hrueggemanni & - & \multirow{3}{*}{ Tanner [21] } \\
\hline & Acropora pnlifera & - & \\
\hline & Pocillopora damicornis & $0 /-$ & \\
\hline
\end{tabular}


Table 2. Cont.

\begin{tabular}{|c|c|c|c|}
\hline Donor Rhodophyta & Target Organism-Annelida & Effect & References \\
\hline Grateloupia turu turu & $\begin{array}{c}\text { Sabellaria cementarium } \\
\text { Spinoidae sp. }\end{array}$ & $\begin{array}{l}- \\
-\end{array}$ & Warkus et al. [41] \\
\hline Polysiphonia denudata & $\begin{array}{l}\text { Sabellaria cementarium } \\
\text { Spinoidae sp. }\end{array}$ & $\begin{array}{l}- \\
-\end{array}$ & Warkus et al. [41] \\
\hline Donor Rhodophyta & Target Organism-Echinodermata & Effect & References \\
\hline Delisea pulchra & Holopneustes purpurascens & + & Williamson et al. [33] \\
\hline Tichocarpus crinitus & Strongylocentrotus intermedius & - & Ishii et al. [36] \\
\hline Donor Rhodophyta & Target Organism-Chordata & Effect & References \\
\hline Acanthophora spicifera & Carassius auratus auratus & $0 /-$ & Alvarez-Hernández et al. [8] \\
\hline Amphiroa beauvoisii & Carassius auratus auratus & $0 /-$ & Alvarez-Hernández et al. [8] \\
\hline Centroceras clavulatum & Carassius auratus auratus & $0 /-$ & Alvarez-Hernández et al. [8] \\
\hline Ceramium nitens & Carassius auratus auratus & $0 /-$ & Alvarez-Hernández et al. [8] \\
\hline Chondria littoralis & Carassius auratus auratus & 0 & Alvarez-Hernández et al. [8] \\
\hline Chondriopsis dasyphylla f. pyrifera & Carassius auratus auratus & - & Alvarez-Hernández et al. [8] \\
\hline Crassiphycus caudatus (Gracilaria caudata) & Carassius auratus auratus & 0 & Alvarez-Hernández et al. [8] \\
\hline Dermonema virens & Carassius auratus auratus & 0 & Alvarez-Hernández et al. [8] \\
\hline Digenea simplex & Carassius auratus auratus & 0 & Alvarez-Hernández et al. [8] \\
\hline Gracilaria cervicornis & Carassius auratus auratus & $0 /-$ & Alvarez-Hernández et al. [8] \\
\hline Gracilaria tikvahiae & Carassius auratus auratus & $0 /-$ & Alvarez-Hernández et al. [8] \\
\hline Grateloupia filicina & Carassius auratus auratus & 0 & Alvarez-Hernández et al. [8] \\
\hline Hypnea musciformis & Carassius auratus auratus & $0 /-$ & Alvarez-Hernández et al. [8] \\
\hline Hypnea spinella & Carassius auratus auratus & $0 /-$ & Alvarez-Hernández et al. [8] \\
\hline Laurencia obtusa & Carassius auratus auratus & $0 /-$ & Alvarez-Hernández et al. [8] \\
\hline Liagora ceranoides & Carassius auratus auratus & $0 /-$ & Alvarez-Hernández et al. [8] \\
\hline Tayloriella dictyurus & Carassius auratus auratus & 0 & Alvarez-Hernández et al. [8] \\
\hline Yuzurua poiteaui var. gemmifera & Carassius auratus auratus & 0 & Alvarez-Hernández et al. [8] \\
\hline
\end{tabular}

Note: - means inhibiting effects, + means stimulating effect, 0 - means lack of effect.

\subsection{The Allelopathic Activity of Brown Algae}

Ochrophyta (Phaeophyceae) were the most frequently studied organisms among all macroalgal phyla in which allelopathic activity on target organisms was confirmed (Table 3). The strong negative impact of brown algae on Cnidaria has been described in detail by Tanner [21], Del Monaco et al. [25], Webster et al. [29], Fong et al. [39], Kuffner et al. [42], Paul et al. [55], and Olsen et al. [56]. Tanner [21] demonstrated that changes in Acropora sp. cover were significantly affected by the presence of this brown algae. Later, Kuffner et al. [42] showed that tested brown algae (Dictyota menstrualis and Lobophora variegata) inhibited recruitment and avoidance behavior in Porites astreoides larvae. Olsen et al. [56] also provided evidence that the presence of the brown alga $D$. menstrualis has direct negative effects on the survival and recruitment of Caribbean coral P. astreoides. Moreover, Webster et al. [29] showed the negative effect of brown algae Sphacelaria sp. on larval settlement and the growth as well as the survival of coral recruits Acropora millepora. Fong et al. [39] demonstrated that mortality of Pocillopora acuta larvae increased considerably with increasing concentrations of Lobophora sp. extracts. Furthermore, Del Monaco et al. [25] shown that elevated $\mathrm{CO}_{2}$ concentrations increased the deleterious effect of Canistrocarpus (=Dictyota) cervicornis on Acropora intermedia. In turn, Paul et al. [55] provided evidence that Dictyota pulchella and Dictyota pinnatifida may adversely affect larval settlements and recruitment. 
Table 3. Examples of allelopathic activity of brown algae against competitors and herbivores.

\begin{tabular}{|c|c|c|c|}
\hline Donor Ochrophyta & Target Organism-Cnidaria & Effect & References \\
\hline \multirow[t]{2}{*}{ Dictyota bartayresiana } & Phialophora verrucosa & - & Longo and Hay [26] \\
\hline & Acropora millepora & - & Rasher et al. [22] \\
\hline \multirow[t]{3}{*}{ Dictyota bartayresiana } & Montipora digitata & - & $\begin{array}{l}\text { Rasher et al. [22], } \\
\text { Ritson-Williams et al. }\end{array}$ \\
\hline & Pocillopora damicornis & - & \\
\hline & Acropora cervicornis & 0 & \\
\hline \multirow[t]{2}{*}{ Dictyota bartayresiana } & Acropora palmata & - & Ritson-Williams et al. [27] \\
\hline & Pseudodiploria strigosa & 0 & \\
\hline Dictyota cervicornis & Acropora intermedia & - & Del Monaco et al. [25] \\
\hline Dictyota menstrualis & Porites astreoides & - & Olsen et al. [56] \\
\hline Dictyota pinnatifida & Porites astreoides & - & Paul et al. [55] \\
\hline \multirow[t]{2}{*}{ Dictyota pulchella } & Porites astreoides & - & Paul et al. [55] \\
\hline & Acropora cervicornis & 0 & \\
\hline \multirow[t]{2}{*}{ Dictyota pulchella } & Acropora palmata & - & Ritson-Williams et al. [27] \\
\hline & Pseudodiploria strigosa & 0 & \\
\hline \multirow{2}{*}{ Dictyota sp. } & Montastraea faveolate & $0 /-$ & \\
\hline & Porites astreoides & $0 /-$ & Morrow et al. [52] \\
\hline \multirow{3}{*}{ Dictyota sp. } & Briareum asbestinum & - & \\
\hline & Porites astreoides & - & Kuffner et al. [42] \\
\hline & Acropora muricate & - & \\
\hline \multirow{4}{*}{ Lobophora abscondita } & Montipora hirsute & 0 & \\
\hline & Porites cylindrica & 0 & Vieira et al. [57] \\
\hline & Stylophora pistillata & - & \\
\hline & Acropora muricate & - & \\
\hline \multirow{4}{*}{ Lobophora crassa } & Montipora hirsute & 0 & \\
\hline & Porites cylindrica & 0 & Vieira et al. [57] \\
\hline & Stylophora pistillata & - & \\
\hline & Acropora muricate & - & \\
\hline \multirow{4}{*}{ Lobophora dimorpha } & Montipora hirsute & 0 & \\
\hline & Porites cylindrica & 0 & Vieira et al. [57] \\
\hline & Stylophora pistillata & - & \\
\hline & Acropora muricate & - & \\
\hline \multirow{4}{*}{ Lobophora hederacea } & Montipora hirsute & 0 & \\
\hline & Porites cylindrica & 0 & Vieira et al. [57] \\
\hline & Stylophora pistillata & - & \\
\hline & Acropora muricate & - & \\
\hline \multirow{4}{*}{ Lobophora monticola } & Montipora hirsute & 0 & \\
\hline & Porites cylindrica & 0 & Vieira et al. [57] \\
\hline & Stylophora pistillata & - & \\
\hline & Acropora muricate & - & \\
\hline \multirow{3}{*}{ Lobophora nigrescens } & Montipora hirsute & 0 & \\
\hline & Porites cylindrica & 0 & Vieira et al. [57] \\
\hline & Stylophora pistillata & - & \\
\hline \multirow{5}{*}{ Lobophora rosacea } & Acropora muricate & - & \\
\hline & Montipora hirsute & 0 & \\
\hline & Porites cylindrica & 0 & Vieira et al. [57] \\
\hline & Stylophora pistillata & - & \\
\hline & Acropora muricate & - & \\
\hline \multirow{3}{*}{ Lobophora undulata } & Montipora hirsute & 0 & \\
\hline & Porites cylindrica & 0 & Vieira et al. [57] \\
\hline & Stylophora pistillata & - & \\
\hline Lobophora variegata & Acropora millepora & + & Birrell et al. [40] \\
\hline \multirow{2}{*}{ Lobophora variegata } & Briareum asbestinum & - & \\
\hline & Porites astreoides & - & Kuffner et al. [42] \\
\hline \multirow{2}{*}{ Lobophora variegata } & Montastraea faveolate & - & \\
\hline & Porites astreoides & - & Morrow et al. [52] \\
\hline Lobophora sp. & Pocillopora acuta & - & Fong et al. [39] \\
\hline & Acropora cervicornis & - & \\
\hline Lobophora sp. & Acropora palmata & - & Ritson-Williams et al. [27] \\
\hline & Pseudodiploria strigosa & 0 & \\
\hline
\end{tabular}


Table 3. Cont.

\begin{tabular}{|c|c|c|c|}
\hline Donor Ochrophyta & Target Organism-Cnidaria & Effect & References \\
\hline \multirow{3}{*}{ Padina boryana } & Acropora millepora & - & \multirow{3}{*}{$\begin{array}{l}\text { Rasher et al. [22], } \\
\text { Ritson-Williams et al. [24] }\end{array}$} \\
\hline & Montipora digitata & 0 & \\
\hline & Pocillopora damicornis & - & \\
\hline Padina minor & Pocillopora damicornis & 0 & \multirow{3}{*}{$\begin{array}{l}\text { Lee et al. [28] } \\
\text { Birrell et al. [40] }\end{array}$} \\
\hline \multirow[t]{2}{*}{ Padina sp. } & Acropora millepora & - & \\
\hline & Acropora millepora & - & \\
\hline \multirow[t]{2}{*}{ Sargassum polycystum } & Montipora digitata & 0 & \multirow[t]{2}{*}{ Ritson-Williams et al. [24] } \\
\hline & Pocillopora damicornis & - & \\
\hline Sargassum sp. & Pocillopora damicornis & - & Lee et al. [28] \\
\hline \multirow[t]{2}{*}{ Sphacelaria sp. } & Acropora millepora & - & \multirow{2}{*}{ Webster et al. [29] } \\
\hline & Acropora millepora & 0 & \\
\hline \multirow{3}{*}{ Turbinaria conoides } & Montipora digitata & 0 & \multirow[t]{3}{*}{ Rasher et al. [22] } \\
\hline & Pocillopora damicornis & 0 & \\
\hline & Acropora millepora & - & \\
\hline \multirow[t]{2}{*}{ Turbinaria conoides } & Montipora digitata & 0 & \multirow[t]{2}{*}{ Ritson-Williams et al. [24] } \\
\hline & Pocillopora damicornis & - & \\
\hline \multirow[t]{2}{*}{ Turbinaria ornata } & Phialophora verrucosa & 0 & \multirow[t]{2}{*}{ Longo and Hay [26] } \\
\hline & Acropora cuneata & - & \\
\hline \multirow{3}{*}{ Turbinaria ornata } & Acropora hrueggemanni & - & \multirow{3}{*}{ Tanner [21] } \\
\hline & Acropora pnlifera & - & \\
\hline & Pocillopora damicornis & - & \\
\hline Donor Ochrophyta & Target Organism-Mollusca & Effect & References \\
\hline Dilophus okamurae & Haliotis discus hannai & - & Suzuki et al. [34] \\
\hline Donor Ochrophyta & Target Organism-Annelida & Effect & References \\
\hline \multirow[b]{2}{*}{ Desmarestia viridis } & Sabellaria cementarium & - & \multirow{2}{*}{ Warkus et al. [41] } \\
\hline & Spinoidae sp. & - & \\
\hline \multirow{2}{*}{ Laminaria sp. } & Sabellaria cementarium & - & \multirow{2}{*}{ Warkus et al. [41] } \\
\hline & Spinoidae sp. & - & \\
\hline Donor Ochrophyta & Target Organism-Echinodermata & Effect & References \\
\hline Dictyota pfaffi & Lytechinus variegates & - & Barbosa et al. [35] \\
\hline Ecklonia radiata & Holopneustes purpurascens & 0 & Williamson et al. [33] \\
\hline Stypopodium zonale & Strongylocentrotus purpuratus & - & Gerwick and Fenical [31] \\
\hline Donor Ochrophyta & Target Organism-Arthropoda & Effect & References \\
\hline Dictyota pfaffii & Pachygrapsus transversus & 0 & Barbosa et al. [35] \\
\hline Donor Ochrophyta & Target Organism-Chordata & Effect & References \\
\hline Dictyopteris delicatula & Carassius auratus auratus & $0 /-$ & Alvarez-Hernández et al. [8] \\
\hline Dictyota bartayresiana & Carassius auratus auratus & $0 /-$ & Alvarez-Hernández et al. [8] \\
\hline Dictyota implexa & Carassius auratus auratus & $0 /-$ & Alvarez-Hernández et al. [8] \\
\hline Dictyota spinulosa & Tilapia mossambica & - & Tanaka and Higa [32] \\
\hline Lobophora variegata & Carassius auratus auratus & $0 /-$ & Alvarez-Hernández et al. [8] \\
\hline Padina gymnospora & Carassius auratus auratus & $0 /-$ & Alvarez-Hernández et al. [8] \\
\hline Sargassum liebmannii & Carassius auratus auratus & $0 /-$ & Alvarez-Hernández et al. [8] \\
\hline Stypopodium zonale & Carassius auratus auratus & $0 /-$ & Alvarez-Hernández et al. [8] \\
\hline Stypopodium zonale & Eupomacentrus leucostictus & - & Gerwick and Fenical [31] \\
\hline Stypopodium zonale & Eupomacentrus leucostictus & - & Gerwick et al. [30] \\
\hline
\end{tabular}

Note: - means inhibiting effects, + means stimulating effect, $0-$ means lack of effect.

Several studies have shown that brown algae can have different effects on animals depending on the donor and target species [22,24,26-28,52,57]. Lee et al. [28] examined the effects of macroalgal species on the settlement success of Pocillopora damicomis larvae under aquarium conditions. Longo and Hay [26] also conducted field experiments assessing the effects of extracts obtained from Dictyota bartayresiana and Turbinaria ornata on the coral Pocillopora vcerrucosa. Ritson-Williams et al. [24] showed that the brown algae 
D. bartayresiana negatively affected Acropora millepora, Montipora digitata, and P. damicornis. Four years later, Ritson-Williams et al. [27] tested settlements in the presence of different algae of three coral species: Acropora palmata, Acropora cervicornis, and Pseudodiploria strigosa. Vieira et al. [57] showed that extracts obtained from Lobophora sp. can bleach certain coral species during direct contact. Furthermore, the authors demonstrated that the studied corals differed in their sensitivity to the presence of an extract obtained from brown algae. In turn, Morrow et al. [52] found that both the crude extracts and the presence of live brown algae induced significant changes in the bacterial complex associated with corals and sublethal stress responses in Montastraea faveolata. Furthermore, Rasher et al. [22] demonstrated that macroalgae can directly cause bleaching and death of corals by the transfer of hydrophobic allelochemicals present on their surfaces. It was found that damage to corals has generally been confined to places where it encounters the macroalgae. However, contact with the corals had no effect on these brown algae species. These findings suggest that the deleterious effects on corals are caused by allelopathic compounds rather than by physical contact. Conversely, Birrell et al. [40] have shown that Ochrophyta (Lobophora variegata) can also have a positive effect on Cnidaria Acropora millepora. To study allelopathic compounds that control seaweed-herbivore interactions, Suzuki et al. [34] investigated the effects of Dilophus okamurae on Mollusca (Haliotis discus hannai). Only Warkus et al. [41] described the negative allelopathic effect of brown algae Desmarestia viridis and Laminaria sp. on polychaeta Sabellaria cementarium and Spinoidae sp. (Annelida). Barbosa et al. [35] showed that compounds obtained from Dictyota pfaffii were effective in inhibiting feeding by the sea urchin Lytechinus variegatus (Echinodermata). Research conducted by Gerwick and Fenical [31] also confirmed the negative effect of Ochrophyta on Echinodermata. Conversely, Williamson et al. [33] showed that Ecklonia radiata had no effect on the development and metamorphosis of Holopneustes purpurascens (Echinodermata) larvae. Barbosa et al. [35] were the only authors who documented that the compound obtained from Dictyota pfaffii did not inhibit feeding by the crab Pachygrapsus transversus (Arthropoda). Research conducted by Alvarez-Hernández et al. [8] showed that brown algae may adversely affect animals belonging to Chordata phylum. Gerwick et al. [30] performed an experiment showing that when Stypopodium zonale was placed in the aquarium, the water became a rust colored and toxic to the herbivorous fish Eupomcentrus leucostictus. Later, Gerwick and Fenical [31] described that nearly all the compounds isolated from S. zonale showed negative effects on the same species of reef-dwelling fish. It has been suggested that the production of noxious and allelopathic substances contributes significantly to the survival of $S$. zonale in predator-rich areas in which it abounds.

All these results indicate that brown algae may affect the marine ecosystem by limiting the development of associated animals. Moreover, recent field assays have suggested the potential role of chemical mediators in this interaction. It has also been suggested that certain brown algae species may produce allelopathic compounds that may play an important ecological function as a defense strategy against herbivores worldwide [35].

\section{Allelopathic Compounds Produced by Macroalgae}

Since there is very little information about the compounds produced by macroalgae, this section provides examples of characterized macroalgae compounds that interact with other heterotrophic organisms (not only competing and herbivorous).

Many studies have reported novel secondary metabolites produced by marine Chlorophyta species, which have significant biological activity on target organisms (Table 4). Capisterones, caulerpals, cycloeudesmol, cymobarbatol, halitunal, isorawsonol, lyengaroside, and sphingosin are compounds that have been isolated from Penicillus capitatus, Caulerpa taxifolia, Chondria oppositiclada, Cymopolia barbat, Halimeda tuna, Arrainvilla rawsonii, Codium iyengarii, and Ulva fasciata green algae, respectively [58]. Dopamine is an allelopathic compound produced by the green algae Ulvaria obscura that negatively affects the development of coexisting aquatic animals [46,59]. The $U$. obscura is a common Chlorophyta that often forms the green tides in the northeastern Pacific [47], where it can 
coexist with other green macroalgal species such as Ulva lactuca, U. prolifera, and U. linza. Nelson et al. [47] hypothesized that dopamine is responsible for some harmful effects observed in coexisting aquatic animals. Paul and Fenical [60] showed that halimedatrial can completely inhibit the motility of sea urchin (Lytechinus pictus) sperm. Halimedatrial is a diterepene trialdhyde isolated from various species of the genus Halimeda (Chlorophyta) such as $H$. tuna, $H$. opuntia, $H$. incrassata, $H$. simulans, $H$. scabra, and $H$. copiosa. This compound is also toxic toward reef damselfishes (Eupomacentrus planifrons and Dascyllus aruanus) and significantly reduces feeding in these herbivorous fishes [60].

Marine red algae are the most important source of many biologically active compounds (Table 4). For instance, the Rhodophyta Callophycus serratus, Plocumium carttilagineum, Portieria hornemanii, Laurencia okamurai, and Laurencia viridis are sources of bromophycolides C-I, furoplocamioid C, halmon, laurinterol, and thyresenol A-B compounds, respectively [58]. Moreover, tichocarpols A and B are compounds isolated from the red alga Tichocarpus crinitus, and they exhibit antifeedant activity against the sea urchin Strongylocentrotus intermedius [36]. Williamson et al. [33] described that the floridoside-isethionic acid complex produced and released by Delisea pulchra induced metamorphosis in the Holopneustes purpurascens sea urchin.

Many bioactive metabolites with different biological activities have also been isolated from Ochrophyta (Table 4). Brown algae species such as Bifurcaria bifurcata, Dictyota dichotoma, Cystoseira tamariscifolia, Lobophora variegate, Sargassum siliquastrum, and Turbinaria ornate can produce compounds such as bifurcadiol, dictyotins, meroditerpenoid, lobophorolide, sargachromanols, and turbinaric acid, respectively [58]. Tanaka and Higa [32] noted that Dictyota spinulosa are not commonly eaten by the herbivorous fish Tilapia mossambica because it produces allelopathic diterpene. Similarly, two diterpenoids (dictyterepenoids A and B), which were isolated from the Dilophus okamurae brown algae, display antifeedant activity against the Haliotis discus hannai abalone [34]. Furthermore, Dictyota pfaffi brown algae also produce antifeedant compounds (diterpenoid 10,18-diacetoxy-8-hydroxy-2,6-dolabelladiene) against herbivores (sea urchins and fishes) [35]. Gerwick et al. [30] showed that stypoldione isolated from Stypopodium zonale brown algae exhibits ichthyotoxic activity on herbivorous reef-dwelling fish Eupomcentrus leucostictus. Two years later, Gerwick and Fenical [31] described other compounds obtained from this brown alga, including stypotriol, stypodiol, epistypodiol, epitaondiol, 2-(geranyl-geranyl)-5-methyl-1,4-benzohydroquinone, 2(geranyl-geranyl)-5-methyl-1,4-benzoquinone, taondiol, and atomaric acid, which showed toxic effects toward E. leucostictus. These authors also reported that stypoldione from $S$. zonale is a potent inhibitor of cell division in the fertilized eggs of the sea urchin Strongylocentrotus purpuratus.

Although freshwater and brackish macroscopic green algae (Chlorophyta and Charophyta) can produce allelochemicals with interesting properties [61-64], they have not been widely investigated (Table 4). Wium-Andersen et al. [61,62] showed that freshwater Chara globularis (Charophyta, Charophyceae) negatively affects natural phytoplankton assemblages via two sulfuric compounds: dithiolane and trithiane. Anthoni et al. [63] isolated charamin, which has strong antibiotic activity, from C. globularis. More recently, Korzeniowska et al. [64] identified nine phenolic compounds obtained from freshwater Cladophora glomerata (Chlorophyta, Ulvophyceae) however, the activity of these compounds on aquatic animals has not been tested (Table 4). 
Table 4. Macroalgae capable of producing bioactive compounds against other heterotrophic organisms (not only competing and herbivorous), location of their environmental occurrence, name of compounds, and their effect on target organisms.

\begin{tabular}{|c|c|c|c|c|}
\hline Phylum/Species & Habitat & Compound & Activity & References \\
\hline \multicolumn{5}{|c|}{ Green Algae (Chlorophyta) } \\
\hline Avrainvillea nigricans & marine & Nigricanosides A-B & $\begin{array}{l}\text { Antimitotic agent } \\
\text { Protein tyrosine }\end{array}$ & Williams et al. [65] \\
\hline Avrainvillea nigricans & marine & Hydroxyisoavrainvilleol & $\begin{array}{c}\text { phosphate 1B inhibitors } \\
\text { (PTP1B) }\end{array}$ & Colon et al. [66] \\
\hline Avrainvillea rawsonii & marine & Isorawsonol & $\begin{array}{c}\text { Cytotoxic and } \\
\text { immunosuppressive } \\
\text { activities }\end{array}$ & Chen et al. [67] \\
\hline Bryopsis sp. & marine & Kahalalide F & $\begin{array}{l}\text { Cytotoxic and } \\
\text { immunosuppressive } \\
\text { activities }\end{array}$ & Hamann and Scheuer [68] \\
\hline Bryopsis sp. & marine & Kahalalide P & $\begin{array}{l}\text { Cytotoxic and } \\
\text { immunosuppressive } \\
\text { activities }\end{array}$ & Dmitrenok et al. [69] \\
\hline Caulerpa racemosa & marine & Sulfoquinovosyldiacylglycerol & Antiviral activity & Wang et al. [70] \\
\hline Caulerpa taxifolia & marine & Caulerpals A-B & Anti-fungal activity & Aguilar-Santos [71] \\
\hline Chara globularis & freshwater & Charamin & Antibiotic activity & Anthoni et al. [63] \\
\hline Chara globularis & freshwater & Dithiolane, Trithiane & Antialgal activity & Wium-Andersen et al. [61] \\
\hline Cladophora glomerata & freshwater & $\begin{array}{c}\text { Gallic acid, Chlorogenic acid, } \\
\text { Syringic acid, } p \text {-coumaric acid, } \\
\text { Myricetin, 3,4-dihydroxybenzoic } \\
\text { acid, Vanillic acid, 4-hydroxybenzoic } \\
\text { acid, Rutin }\end{array}$ & Unknown & Korzeniowska et al. [64] \\
\hline Codium iyengarii & marine & Lyengaroside & Antibacterial activity & Ali et al. [72] \\
\hline Cymopolia barbata & marine & $\begin{array}{l}\text { Cymobarbatol, } \\
\text { 4-isocymobarbatol }\end{array}$ & Antimutagenic activity & Wall et al. [73] \\
\hline $\begin{array}{l}\text { Halimeda tuna, Halimeda } \\
\text { opuntia, Halimeda } \\
\text { incrassata, Halimeda } \\
\text { simulans, Halimeda } \\
\text { scabra, Halimeda copiosa }\end{array}$ & marine & Halimedatrial & $\begin{array}{c}\text { Cytotoxic and } \\
\text { antimicrobial activities }\end{array}$ & Paul and Fenical [60] \\
\hline Halimeda tuna & marine & Halitunal & Antibacterial activity & Koehn et al. [74] \\
\hline Halimeda sp. & marine & Halimedatrial & $\begin{array}{l}\text { Antimicrobial and } \\
\text { cytotoxic properties }\end{array}$ & Paul and Fenical [75] \\
\hline Penicillus capitatus & marine & Capisterones A-B & $\begin{array}{l}\text { Anti-fungal activity } \\
\text { Cytotoxic and }\end{array}$ & Puglisi et al. [76] \\
\hline Tydemania expeditionis & marine & Cycloartenol disulfates & $\begin{array}{l}\text { immunosuppressive } \\
\text { activities }\end{array}$ & Govindan et al. [77] \\
\hline $\begin{array}{l}\text { Ulva }(\text { Enteromorpha }) \\
\quad \text { intestinals }\end{array}$ & marine & Penostatins A-H & $\begin{array}{c}\text { Cytotoxic and } \\
\text { immunosuppressive } \\
\text { activities }\end{array}$ & $\begin{array}{l}\text { Takahashi et al. [78], } \\
\text { Iwamoto et al. [79,80] }\end{array}$ \\
\hline $\begin{array}{l}\text { Ulva (Enteromorpha) } \\
\quad \text { intestinalis }\end{array}$ & marine & Cytochalasans, penochalasins A-H & Cytotoxic activity & Numata et al. [81] \\
\hline $\begin{array}{l}\text { Ulva (Enteromorpha) } \\
\quad \text { intestinalis }\end{array}$ & marine & Chaetoglobosin & Cytotoxic activity & Iwamoto et al. [82] \\
\hline $\begin{array}{l}\text { Ulva (Enteromorpha) } \\
\quad \text { intestinals }\end{array}$ & marine & Communesins A-B & $\begin{array}{c}\text { Cytotoxic and } \\
\text { immunosuppressive } \\
\text { activities }\end{array}$ & Numata et al. [83] \\
\hline Ulva lactuca & marine & $\begin{array}{l}\text { 3-0- } \beta \text {-D-glucopyranosy-lstigmasta- } \\
5,25 \text {-diene }\end{array}$ & $\begin{array}{l}\text { Anti-inflammatory } \\
\text { substances }\end{array}$ & Awad et al. [84] \\
\hline Ulvaria obscura & marine & Dopamine & $\begin{array}{l}\text { Feeding-deterrent } \\
\text { substances }\end{array}$ & $\begin{array}{l}\text { Tocher and Craigie [59], } \\
\text { Van Alstyne et al. [46] }\end{array}$ \\
\hline \multicolumn{5}{|c|}{ Red Algae (Rhodophyta) } \\
\hline $\begin{array}{l}\text { Beckerella (Gelidium) } \\
\text { subcostatum }\end{array}$ & marine & $\begin{array}{l}\text { Bromo- beckerelide, epimer, } \\
\text { chlorobeckerelide }\end{array}$ & Antimicrobial activity & Ohta [85] \\
\hline Callophycus serratus & marine & Bromophycolides A-B & Cytotoxic activity & Kubanek et al. [86] \\
\hline Callophycus serratus & marine & Bromophycolides C-I & Cytotoxic activity & Kubanek et al. [87] \\
\hline Callophycus serratus & marine & $\begin{array}{l}\text { Callophycoic acids A-H, } \\
\text { diterpene-phenols, callophycols A-B }\end{array}$ & $\begin{array}{c}\text { Antibacterial, antimalarial, } \\
\text { anti-tumor and antifungal } \\
\text { activity }\end{array}$ & Lane et al. [88] \\
\hline Chondria armata & marine & Isodomic acid A-C & Insecticidal activity & Maeda et al. [89] \\
\hline Chondria atropurpurea & marine & Chondriamide C, 3-indolacrylamide & Anthelmintic activity & Davyt et al. [90] \\
\hline Chondria oppositiclada & marine & Cycloeudesmol & Antibacterial activity & Fenical and Sims [91] \\
\hline Delisea pulchra & marine & Floridoside-isethionic acid complex & $\begin{array}{l}\text { Induction of animal } \\
\text { metamorphosis }\end{array}$ & Williamson et al. [33] \\
\hline
\end{tabular}


Table 4. Cont.

\begin{tabular}{|c|c|c|c|c|}
\hline Phylum/Species & Habitat & Compound & Activity & References \\
\hline \multicolumn{5}{|c|}{ Red Algae (Rhodophyta) } \\
\hline Digenea simplex & marine & $\alpha$-alko-kainic acid & $\begin{array}{l}\text { Neurophysiological } \\
\text { activity }\end{array}$ & $\begin{array}{c}\text { Biscoe et al. [92], Ferkany and } \\
\text { Coyle [93] }\end{array}$ \\
\hline Gracilaria asiatica & marine & $\begin{array}{c}\text { Cerebroside gracilarioside, } \\
\text { ceramides gracilamides A-B }\end{array}$ & Cytotoxic activity & Sun et al. [94] \\
\hline $\begin{array}{l}\text { Gigartina tenella } \\
\text { Jania rubens }\end{array}$ & $\begin{array}{l}\text { marine } \\
\text { marine }\end{array}$ & $\begin{array}{l}\text { Sulquinovosyldiacylglycerol: KM043 } \\
\text { Deoxyparguerol-7-acetate }\end{array}$ & $\begin{array}{l}\text { Antiviral activity } \\
\text { Anthelmintic activity }\end{array}$ & $\begin{array}{l}\text { Ohata et al. [95] } \\
\text { Awad [96] }\end{array}$ \\
\hline Laurencia brongniartii & marine & Polybromoindoles & $\begin{array}{l}\text { Antimicrobial activity, } \\
\text { cytotoxic activity }\end{array}$ & $\begin{array}{l}\text { Carter et al. [97], } \\
\text { El Gamal et al. [98] }\end{array}$ \\
\hline Laurencia brongniartii & marine & Brominated indoles & Antibacterial activities & Carter et al. [97] \\
\hline Laurencia elata & marine & Elatol & Antibacterial activities & Sims [99] \\
\hline Laurencia obtusa & marine & Teurilene, thyrsiferyl 23-acetate & Cytotoxic activity & Suzuki et al. [100] \\
\hline Laurencia obtusa & marine & $\begin{array}{l}\text { 3,7-dihydroxydihydrolaurene, } \\
\text { perforenol B }\end{array}$ & Cytotoxic activity & Kladi et al. [101] \\
\hline Laurencia obtusa & marine & Neorogioldiol B, prevezol B-D & Cytotoxic activity & IIopoulou et al. [102] \\
\hline Laurencia obtusa & marine & Iso-obtusol & Antibacterial activities & Gonzalez et al. [103,104] \\
\hline Laurencia obtusa & marine & Sesquiterpene & Antimalarial activity & Topeu et al. [105] \\
\hline Laurencia pinnatifida & marine & $\begin{array}{c}\text { Dehydrothyrsiferol, thyresenol A } \\
\text { and B }\end{array}$ & Cytotoxic activity & Norte et al. [106], Pec et al. [107] \\
\hline Laurancia pinnata & marine & $\begin{array}{c}\text { Laurepinacine, isolaurepinnacin } \\
\text { Brominated diterpene, }\end{array}$ & Insecticidal activity & Fukuzawa and Masamune [108] \\
\hline Laurencia mariannensis & marine & $\begin{array}{l}\text { 10-hydroxykahukuene B, } \\
\text { 9-deoxyelatol, isoda-ctyloxene A, } \\
\text { C15-acetogenin, laurenmariallene, } \\
\text { sesquiterpenes }\end{array}$ & Antibacterial activities & Gonzalez et al. [109] \\
\hline Laurencia nidifica & marine & $\begin{array}{l}\text { Laurinterol, isolaurinterol, aplysin, } \\
\alpha \text {-bromocuparene }\end{array}$ & $\begin{array}{l}\text { Insecticidal and repellent } \\
\text { activities }\end{array}$ & Ishii et al. [110] \\
\hline Laurencia nipponica & marine & $\begin{array}{c}\text { (Z)-Laureatin, (Z)-isolaureatin, } \\
\text { deoxyprepacifenol }\end{array}$ & Insecticidal activity & $\begin{array}{l}\text { Watanabe et al. [111], } \\
\text { El Sayed et al. [112] }\end{array}$ \\
\hline Laurencia okamurae & marine & Laurinterol & Cytotoxic activity & Moon-Moo et al. [113] \\
\hline Laurencia scoparia & marine & $\beta$-bisabolene sesquiterpenes & Anthelmintic activity & Davyt et al. [114] \\
\hline Laurencia tristicha & marine & $\begin{array}{l}\text { Cholest-5-en-3 } \beta, 7 \alpha \text {-diol } \\
\text { Debromoepiaplysinol }\end{array}$ & Cytotoxic activity & Sun et al. [115] \\
\hline Laurencia venusta & marine & Venustatriol & Antiviral activity & Sakemi et al. [116] \\
\hline Laurencia yonaguniensis & marine & Neoirietetraol & Cytotoxic activity & Takahashi et al. [117] \\
\hline Lophocladia sp. & marine & Lophocladine B & Cytotoxic activity & Gross et al. [118] \\
\hline Murrayella periclados & marine & 12S-hydroxyeicosapentaenoic acid & Lipooxygenase inhibitor & Bernari and Gerwick [119] \\
\hline Odonthalia corymbifera & marine & Bromophenols & $\begin{array}{l}\text { Inhibition of isocitrate } \\
\text { lyase enzyme }\end{array}$ & Lee et al. [120] \\
\hline Peyssonnelia sp. & marine & Avarol & Antiviral activity & Talpir et al. [121] \\
\hline Plocamium corallorhiza & marine & Plocaralides B-C & Cytotoxic activity & Knott et al. [122] \\
\hline Plocamium telfairiae & marine & Telfairine & Insecticidal activity & Watanabe et al. [123] \\
\hline Ptilota filicina & marine & Ptiollodene & Lipo-oxygenase inhibitor & Lopez and Gerwick [124] \\
\hline Symphyocladia latiuscula & marine & Tasipeptins A-B & $\begin{array}{l}\text { Aldose reductase } \\
\text { inhibitors activity }\end{array}$ & Wang et al. [125] \\
\hline Vidalia obtusiloba & marine & Vidalols A-B & $\begin{array}{l}\text { Anti-inflammatory } \\
\text { activity }\end{array}$ & Wiemer et al. [126] \\
\hline \multicolumn{5}{|c|}{ Brown Algae (Ochrophyta) } \\
\hline Chondria oppositiclada & marine & Cycloeudesmol & Antibacterial activity & Fenical and Sims [91] \\
\hline Cystoseira crinita & marine & Meroterpenoids & $\begin{array}{l}\text { Free radical scavenger and } \\
\text { antioxidant activities }\end{array}$ & Fisch et al. [127] \\
\hline Cystoseira myrica & marine & Hydroazulene diterpenes & Cytotoxic activity & Ayyad et al. [128] \\
\hline Cystoseira tamariscifolia & marine & Methoxybifurcarenone & $\begin{array}{l}\text { Antifungal and } \\
\text { antibacterial activity }\end{array}$ & Bennamara et al. [129] \\
\hline Cystophora siliquosa & marine & Cystophorene & $\begin{array}{l}\text { Sperm-attractants } \\
\text { pheromone }\end{array}$ & Muller et al. [130] \\
\hline Dictyopteris undulata & marine & Yahazunol & Antimicrobial activity & Ochi et al. [131] \\
\hline Dictyopteris undulata & marine & Cyclozonarone & Feeding-deterrent activity & Kurata et al. [132] \\
\hline Dictyopteris zonarioides & marine & Zonarol, isozonarol & Antifungal activity & Fenical et al. [133] \\
\hline Dictyota pfaffi & marine & $\begin{array}{l}\text { 10,18-diacetoxy-8-hydroxy } \\
\text { 2,6-dollabeladiene (dolabellane 1) }\end{array}$ & Antiviral activity & Barbosa et al. $[35,134]$ \\
\hline Dictyota spinulosa & marine & Hydroxydictyodial & $\begin{array}{l}\text { Feeding-deterrent } \\
\text { substances }\end{array}$ & Tanaka and Higa [32] \\
\hline Dictyota sp. & marine & Dolabellane diterpenes & Cytotoxic activity & Tringali et al. [135] \\
\hline Dilophus okamurae & marine & Dictyterepenoids A-B & Antifeedent activity & Suzuki et al. [34] \\
\hline Ecklonia cava & marine & Fucodiphlorethol G & Antioxidant activity & Ham et al. [136] \\
\hline
\end{tabular}


Table 4. Cont.

\begin{tabular}{|c|c|c|c|c|}
\hline Phylum/Species & Habitat & Compound & Activity & References \\
\hline \multicolumn{5}{|c|}{ Brown Algae (Ochrophyta) } \\
\hline Ecklonia stolonifera & marine & $\begin{array}{l}\text { Phloroglucinol, eckstolonol, eckol, } \\
\text { phlorofucofuroeckol A, dieckol }\end{array}$ & Hepatoprotective activity & Kang et al. [137] \\
\hline Giffordia mitchelliae & marine & Giffordene & $\begin{array}{c}\text { Gamete-attracting } \\
\text { pheromone }\end{array}$ & Boland et al. [138] \\
\hline Hizikia fusiformis & marine & Arsenic-containing ribofuranosides & Cytotoxic activity & Edmonds et al. [139] \\
\hline Hormosira banksii & marine & Hormosirene & $\begin{array}{c}\text { Sperm-attractants } \\
\text { pheromone }\end{array}$ & Muller et al. [130] \\
\hline Leptosphaeria sp. & marine & Leptosins M, MI, N, N1 & Cytotoxic activity & Yamada et al. [140] \\
\hline Lobophora variegata & marine & Lobophorolide & Antifungal activity & Kubanek et al. [141] \\
\hline Notheia anomala & marine & cis dihydroxyte-trahydrofuran & Nematocidal activity & Capon et al. [142] \\
\hline Osmundaria serrata & marine & Lanosol enol ether & $\begin{array}{c}\text { Antifungal and } \\
\text { antibacterial activity }\end{array}$ & Barreto and Meyer [143] \\
\hline Perithalia caudata & marine & Caudoxirene & $\begin{array}{c}\text { Gamete-releasing, } \\
\text { gamete-attracting } \\
\text { pheromone }\end{array}$ & Muller et al. [144] \\
\hline Pelvetia siliquosa & marine & Fucosterol & Anti-diabetic activity & Lee et al. [145] \\
\hline Sargassum siliquastrum & marine & Sargachromanols A-P & Antioxidant activity & Jang et al. [146] \\
\hline Sargassum tortile & marine & Dihydroxysargaquinone & Cytotoxic activity & Numata et al. [147] \\
\hline Sargassum tortile & marine & Hydroxysargaquinone, sargasal-I-II & Cytotoxic activity & Numata et al. [148] \\
\hline Sargassum thunbergii & marine & Thunbergols A-B & $\begin{array}{l}\text { Scavenging activities, } \\
\text { antioxidant activity }\end{array}$ & Seo et al. [149] \\
\hline Sargassum thunbergii & marine & Sargothunbergol A & Antioxidant activity & Seo et al. [150] \\
\hline Sargassum thunbergii & marine & Diacylglycerols & Antifungal activity & Kim et al. [151] \\
\hline Stypopodium flabelliforme & marine & Isoepitaondiol & Insecticidal activity & Rovirosa et al. [152] \\
\hline Stypopodium zonale & marine & Stypolactone & Cytotoxic activity & Dorta et al. [153] \\
\hline Stypopodium zonale & marine & Stypotriol, stypoldione & Ichthyotoxic activity & Gerwick et al. [30] \\
\hline Stypopodium zonale & marine & $\begin{array}{c}\text { Stypoquinonic acid, taondiol, } \\
\text { atomaric acid }\end{array}$ & Antimicrobial activity & Wessels et al. [154] \\
\hline Stypopodium zonale & marine & $\begin{array}{l}\text { Stypoldione, stypotriol, stypodiol, } \\
\text { epistypodiol, epitaondiol }\end{array}$ & $\begin{array}{l}\text { Ichthyotoxic activity, } \\
\text { cytotoxic activity }\end{array}$ & Gerwick and Fenical [31] \\
\hline Taonia atomaria & marine & Taondiol & $\begin{array}{l}\text { Antimicrobial activity, } \\
\text { cytotoxic activity }\end{array}$ & Othmani et al. [155] \\
\hline Taonia atomaria & marine & $\begin{array}{l}\text { Tetraprenyl benzoquinone } \\
\text { sargaquinone }\end{array}$ & $\begin{array}{l}\text { Anti-inflammatory } \\
\text { activity }\end{array}$ & Tziveleka et al. [156] \\
\hline Taonia atomaria & marine & Meroditerpenes atomarianones A-B & Cytotoxic activity & Abatis et al. [157] \\
\hline Turbinaria ornata & marine & Turbinaric acid & Cytotoxic activity & Asari et al. [158] \\
\hline
\end{tabular}

Allelopathic activity is likely to involve more than one mechanism. Allelochemicals may indirectly influence multiple physiological processes, and phenotypic reactions to a particular compound may result from secondary effects [159]. Different mechanisms function depending on whether allelopathy occurs in open water (pelagic zone) or is associated with substrate (benthic habitats) [12], and many biotic and abiotic factors influence the severity of allelopathic interactions. Macroalgae secrete allelochemicals by direct contact or through masses of water; this is especially facilitated due to the small molecules that make up these compounds. In the case of direct contact, this happens through compounds contained in epidermal glands, secretory trichomes, or in other ways associated with the plant surface [20]. Allelopathic compounds can alter the permeability and fluidity of cell membranes and disturb the activity of membrane proteins and intracellular enzymes, particularly those that build antioxidant systems [160]. Moreover, allelochemicals can also cause oxidative damage and activation of antioxidant mechanisms [161]. In addition, allelopathic compounds have been observed to affect photosynthesis [162] and have been influenced by environmental factors (temperature, light intensity, water availability, $\mathrm{CO}_{2}$ concentration, and microorganisms) [163]. A potential site of action for allelochemicals is the mitochondria because mitochondrial respiration is essential for the production of ATP, which is used in metabolic processes, for example, macromolecular synthesis [164].

Macroalgae are a rich source of highly bioactive secondary metabolites that may have potential applications. Macroalgae biomass are widely used in the chemical, food, agriculture, cosmetics, pharmacy, and medicine industries. Macroalgae are also rich in various biologically active substances valued for their, among others, antimicrobial, 
anti-inflammatory, antioxidant, antifungal, cytotoxic, and insecticidal activity $[58,165]$. Additionally, allelochemicals from macroalgae on herbivores may have potential in limiting the negative expansion of invasive species worldwide (Table 4). This research highlights the possibility of exploiting the allelopathic potential of macroalgae in commercial aquaculture. The characterization of macroalgal allelochemicals as well as their mode of action are still poorly understood. In addition, most studies have focused on the activity of allelopathic compounds derived from marine macroalgae. Therefore, future research should also include the isolation and identification of allelopathic compounds from freshwater and brackish macroalgae.

\section{The Limitation of Macroalgae-Herbivores Interactions}

Herbivores have a great influence on macroalgae in all water types [166]. A multidisciplinary ecophysiological approach is required to study macroalgae-herbivores interactions in combination with other mechanisms affecting plants. Most macroalgae show some form of anti-herbivore strategy. These relate to physical features that allow escape or chemical features that allow for defense, e.g., by release of secondary metabolites [167]. Thus, research can include both the ecological and molecular levels. The production of allelochemicals has been shown to increase under certain conditions. Del Monaco et al. [25] suggested that increasing ocean acidification can cause advantages to seaweeds over corals and that ocean acidification may enhance the allelopathy of certain macroalgae. Conversely, Ritson-Williams et al. [24] described that increased seawater temperatures made larvae more susceptible to a concurrent local stressor disrupting a key process of coral reef recovery and resilience. The process of synthesizing molecules of allelopathic compounds is controlled by a number of physiological, chemical, and spatial-temporal variables [8]. The toxicity gradient may be related to habitat complexity. More toxic macroalgae extracts are found in reef sites and in rocky intertidal environments. The presence or absence of toxicity was also observed depending on sample collection site and climate [8]. Additionally, allelopathy can only be effective when plants are under stress caused by other mechanisms, such as deprivation of water or intense competition for both nutrients or light. The target plant is also more susceptible to phytotoxins when under stress [168]. Furthermore, bacteria associated with the target or donor organism may metabolize the excreted allelochemicals [12]. It is important to pay attention and avoid misunderstandings, especially in distinguishing allelopathy from any other competitive or noncompetitive relationship [12]. A small number of authors model allelopathic interactions using field or experimental data e.g., [169-174]. Such studies usually must oversimplify processes, which may not always be satisfactory. Thus, the method for testing the effects of allelopathic macroalgae on target organisms should be chosen carefully. Macroalgae extracts and exudates provide an environment that is distant from the environmental conditions of the test organisms while experiments in mesocosms or arranged co-culturing experiments are closer to the conditions of natural occurrence of macroalgae and studied animals and are thus more reflective of naturally occurring processes.

\section{Conclusions}

Macroalgae are the sources of many harmful allelopathic compounds, which are synthesized as a defense strategy against competitors and predators. Macroalgae can produce inhibitory compounds affecting competitors for the Cnidaria phylum on tropical reefs. The strongest negative effect against Cnidaria occur from macroalgae of the genus Bryopsis, Chlorodesmis, Halimeda, and Rhiphilia (Chlorophyta, green algae); Amansia, Asparagopsis, Callophycus, Endosiphonia, Galaxaura, Phacelocarpus, and Plocamium (Rhodophyta, red algae); as well as Sphacelaria (Ochrophyta, brown algae). Several studies have also demonstrated the negative effects of macroalgae on predators (Mollusca, Annelida, Echinodermata, Arthropoda, and Chordata species) upon ingestion. Chaetomorpha, Codium, and Ulva (green algae); Grateloupia and Polysiphonia (red algae); and Desmarestia and Laminaria (brown algae) strongly inhibit Annelida development. Furthermore, red (Tichocarpus sp.) 
and brown (Dictyota sp. and Stypopodium sp.) algae negatively affect species belonging to Echinodermata. Some studies also examined negative effects of Ulvaria obscura (green algae) on Arthropoda species. The strong negative influence of the red algae Chondriopsis sp. on Chordata, and brown algae Dilophus sp. on Mollusca has been demonstrated. Although the term macroalgal allelopathy refers to the effects of substances produced by macroalgae that can be both harmful and beneficial to target organisms, positive effects of algae on aquatic animals are extremely rare. Only certain species of green (Chlorodesmis sp., Ulva sp., and Ulvaria sp.), red (Delisea sp.), and brown algae (Lobophora sp.) positively affect certain Cnidaria, Mollusca, and Echinodermata species. In addition, the allelopathic activity of macroalgae can change according to the taxonomic position of the donor and target organisms, as well as their habitat. However, most studies have focused on the allelopathic effects of macroalgae in marine environments. Therefore, future studies should consider the nature of released substances and their effect on target organisms of freshwater and brackish macroalgae. Furthermore, the allelopathy phenomenon of macroalgae in aquatic ecosystems should be further studied considering both scientific and commercial aspects.

Supplementary Materials: The following are available online at https:/ /www.mdpi.com/article/10 $.3390 /$ ijms22157865/s1.

Author Contributions: Conceptualization, G.B. and S.Ś-W.; methodology, G.B. and S.Ś.-W.; formal analysis, G.B. and S.S.-W.; investigation, G.B. and S.Ś.-W.; resources, K.W., A.W. and I.B.; data curation, K.W., A.W. and I.B.; writing—original draft preparation, G.B., S.Ś.-W., K.W., A.W., I.B., A.L. and J.M.W.; visualization, G.B., S.S.-W., K.W. and A.W.; supervision, A.L. and J.M.W. All authors have read and agreed to the published version of the manuscript.

Funding: This research was funded by the National Science Centre project, grant number 2019/33/N/ ST10/00585, National Science Centre project, grant number 2015/17/B/NZ8/02473, and UGrants-start, grant number 533-O000-GS12-21. The APC was funded by UGrants-start, no. 533-O000-GS12-21.

Institutional Review Board Statement: Not applicable.

Informed Consent Statement: Not applicable.

Data Availability Statement: All data are presented in the article and Supplementary Materials.

Acknowledgments: The authors would like to thank the editor and anonymous reviewers for their valuable comments and suggestions to improve the quality of the paper. The authors gratefully acknowledge the World Bank for providing information on aquaculture production from the website (https:/ / data.worldbank.org/indicator/ER.FSH.AQUA.MT, accessed on 19 June 2021) used in this publication.

Conflicts of Interest: The authors declare no conflict of interest. The funders had no role in the design of the study, in the collection, analyses, or interpretation of data, in the writing of the manuscript, or in the decision to publish the results.

\section{References}

1. Toufique, K.A.; Belton, B. Is Aquaculture Pro-Poor? Empirical Evidence of Impacts on Fish Consumption in Bangladesh. World Dev. 2014, 64, 609-620. [CrossRef]

2. Subasinghe, R. World aquaculture 2015: A brief overview; Food and Agriculture Organization of the United Nations: Rome, Italy, 2017.

3. Van Ginneken, V.; de Vries, E. Towards a Seaweed Based Economy: The Global Ten Billion People Issue at the Midst of the 21st Century. J. FisheriesSciences. Com 2016, 10, 001-011.

4. Oyinlola, M.A.; Reygondeau, G.; Wabnitz, C.C.; Troell, M.; Cheung, W.W. Global estimation of areas with suitable environmental conditions for mariculture species. PLoS ONE 2018, 13, e0191086. [CrossRef]

5. Ben-Ari, T.; Neori, A.; Ben-Ezra, D.; Shauli, L.; Odintsov, V.; Shpigel, M. Management of Ulva lactuca as a biofilter of mariculture effluents in IMTA system. Aquaculture 2014, 434, 493-498. [CrossRef]

6. Hurd, C.L. Seaweed Ecology and Physiology, 2nd ed.; Cambridge University Press: Cambridge, UK, 2015.

7. Carl, C.; de Nys, R.; Paul, N.A. The seeding and cultivation of a tropical species of filamentous Ulva for algal biomass production. PLoS ONE 2014, 9, e98700. [CrossRef] [PubMed]

8. Alvarez-Hernández, S.; Lozano-Ramírez, C.; Rodríguez-Palacio, M. Influence of the Habitat on Marine Macroalgae Toxicity. Annu. Res. Rev. 2019, 33, 1-9. [CrossRef]

9. Harlin, M.M.; Rice, E.L. Allelochemistry in marine macroalgae. Crit. Rev. Plant Sci. 1987, 5, 237-249. [CrossRef] 
10. Lewis, W.M., Jr. Evolutionary interpretations of allelochemical interactions in phytoplankton algae. Am. Nat. 1986, 127, 184-194. [CrossRef]

11. Kersen, P. Red Seaweeds Furcellaria Lumbricalis and Coccotylus Truncatus: Community Structure, Dynamics and Growth in the Northern Baltic Sea. Ph.D. Thesis, Tallinn University, Tallinn, Estonia, 2013.

12. Gross, E.M. Allelopathy of aquatic autotrophs. Crit. Rev. Plant Sci. 2003, 22, 313-339. [CrossRef]

13. Zhu, X.; Dao, G.; Tao, Y.; Zhan, X.; Hu, H. A review on control of harmful algal blooms by plant-derived allelochemicals. J. Hazard. Mater. 2020, 401, 123403. [CrossRef]

14. Rybak, A.S. Ecological preferences of freshwater Ulva flexuosa (Ulvales; Ulvophyceae): Development of macroalgal mats in a Tulce fishpond (Wielkopolska Region, Poland). Oceanol. Hydrobiol. Stud. 2016, 45, 100-111. [CrossRef]

15. Rybak, A.S. Freshwater macroalga, Ulva pilifera (Ulvaceae, Chlorophyta) as an indicator of the trophic state of waters for small water bodies. Ecol. Indic. 2021, 121, 106951. [CrossRef]

16. Rybak, A.S.; Gabka, M. The influence of abiotic factors on the bloom-forming alga Ulva flexuosa (Ulvaceae, Chlorophyta): Possibilities for the control of the green tides in freshwater ecosystems. J. Appl. Phycol. 2018, 30, 1405-1416. [CrossRef]

17. Budzałek, G.; Śliwińska-Wilczewska, S.; Latała, A. Allelopathic effect of Ulva intestinalis L. on the Baltic filamentous cyanobacterium Nostoc sp. AUPC Studia Naturae 2018, 262, 80-89.

18. Złoch, I.; Śliwińska-Wilczewska, S.; Kucharska, M.; Kozłowska, W. Allelopathic effects of Chara species (C. aspera, C. baltica, and C. canescens) on the bloom-forming picocyanobacterium Synechococcus sp. Environ. Sci. Pollut. Res. 2018, 25, 36403-36411. [CrossRef]

19. Guiry, M.D.; Guiry, G.M. AlgaeBase World-Wide Electronic Publication. National University of Ireland: Galway, Ireland. Available online: algaebase.org (accessed on 3 November 2020).

20. Gomes, M.P.; Garcia, Q.S.; Barreto, L.C.; Pimenta, L.P.S.; Matheus, M.T.; Figueredo, C.C. Allelopathy: An overview from micro-to macroscopic organisms, from cells to environments, and the perspectives in a climate-changing world. Biologia 2017, 72, 113-129. [CrossRef]

21. Tanner, J.E. Competition between scleractinian corals and macroalgae: An experimental investigation of coral growth, survival and reproduction. J. Exp. Mar. Biol. Ecol. 1995, 190, 151-168. [CrossRef]

22. Rasher, D.B.; Stout, E.P.; Engel, S.; Kubanek, J.; Hay, M.E. Macroalgal terpenes function as allelopathic agents against reef corals. Proc. Natl. Acad. Sci. USA 2011, 108, 17726-17731. [CrossRef]

23. Bonaldo, R.M.; Hay, M.E. Seaweed-coral interactions: Variance in seaweed allelopathy, coral susceptibility, and potential effects on coral resilience. PLoS ONE 2014, 9, e85786. [CrossRef] [PubMed]

24. Ritson-Williams, R.; Ross, C.; Paul, V.J. Elevated temperature and allelopathy impact coral recruitment. PLoS ONE 2016, 11, e0166581. [CrossRef]

25. Del Monaco, C.; Hay, M.E.; Gartrell, P.; Mumby, P.J.; Diaz-Pulido, G. Effects of ocean acidification on the potency of macroalgal allelopathy to a common coral. Sci. Rep. 2017, 7, 998-1008. [CrossRef] [PubMed]

26. Longo, G.O.; Hay, M.E. Seaweed allelopathy to corals: Are active compounds on, or in, seaweeds? Coral Reefs 2017, 36, 247-253 [CrossRef]

27. Ritson-Williams, R.; Arnold, S.N.; Paul, V.J. The impact of macroalgae and cyanobacteria on larval survival and settlement of the scleractinian corals Acropora palmata, A. cervicornis and Pseudodiploria strigosa. Mar. Biol. 2020, 167, 31. [CrossRef]

28. Lee, C.S.; Walford, J.; Goh, B.P.L. The effect of benthic macroalgae on coral settlement. In Contributions to Marine Science: A Commemorative Volume Celebrating 10 Years of Research on St John's Island; National University of Singapore: Singapore, 2012; pp. 89-93.

29. Webster, F.J.; Babcock, R.C.; Van Keulen, M.; Loneragan, N.R. Macroalgae inhibits larval settlement and increases recruit mortality at Ningaloo Reef, Western Australia. PLoS ONE 2015, 10, e0124162. [CrossRef]

30. Gerwick, W.H.; Fenical, W.; Fritsch, N.; Clardy, J. Stypotriol and stypoldione; ichthyotoxins of mixed biogenesis from the marine alga Stypodium zonale. Tetrahedron Lett. 1979, 2, 145-148. [CrossRef]

31. Gerwick, W.H.; Fenical, W. Ichthyotoxic and cytotoxic metabolites of the tropical brown alga Stypopodium zonale (Lamouroux) Papenfuss. J. Org. Chem. 1981, 46, 22-27. [CrossRef]

32. Tanaka, I.; Higa, T. Hydroxydictyodial, a new antifeedant diterpene from the brown alga Dictyota spinulosa. Chem. Lett. 1984, 2 , 231-232. [CrossRef]

33. Williamson, J.E.; De Nys, R.; Kumar, N.; Carson, D.G.; Steinberg, P.D. Induction of metamorphosis in the sea urchin Holopneustes purpurascens by a metabolite complex from the algal host Delisea pulchra. Biol. Bull. 2000, 198, 332-345. [CrossRef]

34. Suzuki, M.; Yamada, H.; Kurata, K. Dictyterpenoids A and B two novel diterpenoids with feeding-deterrent activity from the brown alga Dilophus okamurae. J. Nat. Prod. 2002, 65, 121-125. [CrossRef]

35. Barbosa, J.P.; Teixeira, V.L.; Pereira, R.C. A dolabellane diterpene from the brown alga Dictyota pfaffii as chemical defense against herbivores. Bot. Marina. 2004, 47, 147-151. [CrossRef]

36. Ishii, T.; Okino, T.; Suzuki, M.; Machiguchi, Y. Tichocarpols A and B, Two Novel Phenylpropanoids with Feeding-Deterrent Activity from the Red Alga Tichocarpus c rinitus. J. Nat. Prod. 2004, 67, 1764-1766. [CrossRef]

37. Morrow, K.M.; Bromhall, K.; Motti, C.A.; Munn, C.B.; Bourne, D.G. Allelochemicals produced by brown macroalgae of the Lobophora genus are active against coral larvae and associated bacteria, supporting pathogenic shifts to Vibrio dominance. Appl. Environ. Microbiol. 2017, 83, e02391-16. [CrossRef]

38. Green-Gavrielidis, L.A.; MacKechnie, F.; Thornber, C.S.; Gomez-Chiarri, M. Bloom-forming macroalgae (Ulva spp.) inhibit the growth of co-occurring macroalgae and decrease eastern oyster larval survival. Mar. Ecol. Prog. Ser. 2018, 595, 27-37. [CrossRef] 
39. Fong, J.; Lim, Z.W.; Bauman, A.G.; Valiyaveettil, S.; Liao, L.M.; Yip, Z.T.; Todd, P.A. Allelopathic effects of macroalgae on Pocillopora acuta coral larvae. Mar. Environ. Res. 2019, 151, 104745. [CrossRef]

40. Birrell, C.L.; McCook, L.J.; Willis, B.L.; Harrington, L. Chemical effects of macroalgae on larval settlement of the broadcast spawning coral Acropora millepora. Mar. Ecol. Prog. Ser. 2008, 362, 129-137.

41. Warkus, E.L.; Wagstaff, M.; Morello, S.; Etter, R. Do Macroalgae Use Allelochemicals to Outcompete Invertebrates for Space in the Gulf of Maine? (Conference Material). 2010. Available online: https://cbs.asu.edu/sites/default/files/warkus_poster.pdf (accessed on 19 June 2021).

42. Kuffner, I.B.; Walters, L.J.; Becerro, M.A.; Paul, V.J.; Ritson-Williams, R.; Beach, K.S. Inhibition of coral recruitment by macroalgae and cyanobacteria. Mar. Ecol. Prog. Ser. 2006, 323, 107-117. [CrossRef]

43. Rasher, D.B.; Hay, M.E. Seaweed allelopathy degrades the resilience and function of coral reefs. Commun. Integr. Biol. 2010, 3, 564-566. [CrossRef]

44. Chadwick, N.E.; Morrow, K.M. Competition among sessile organisms on coral reefs. In Coral Reefs: An Ecosystem in Transition; Springer: Dordrecht, The Netherlands, 2011; pp. 327-371.

45. Roff, G.; Mumby, P.J. Global disparity in the resilience of coral reefs. Trends Ecol. Evol. 2012, 27, 404-413. [CrossRef]

46. Van Alstyne, K.L.; Harvey, E.L.; Cataldo, M. Effects of dopamine, a compound released by the green-tide macroalga Ulvaria obscura (Chlorophyta), on marine algae and invertebrate larvae and juveniles. Phycologia 2014, 53, 195-202. [CrossRef]

47. Nelson, T.A.; Lee, D.J.; Smith, B.C. Are "green tides" harmful algal blooms? Toxic properties of water-soluble extracts from two bloom-forming macroalgae, Ulva fenestrata and Ulvaria obscura (Ulvophyceae). J. Phycol. 2003, 39, 874-879. [CrossRef]

48. Nelson, T.A.; Gregg, B.C. Determination of EC50 for normal oyster larval development in extracts from bloom-forming green seaweeds. Nautilus 2013, 127, 156-159.

49. Huggett, M.J.; De Nys, R.; Williamson, J.E.; Heasman, M.; Steinberg, P.D. Settlement of larval blacklip abalone, Haliotis rubra, in response to green and red macroalgae. Mar. Biol. 2005, 147, 1155-1163. [CrossRef]

50. Muñoz, P.; Ambler, R.; Bulboa, C. Settlement, Survival, and Post-Larval Growth of Red Abalone, Haliotis rufescens, on Polycarbonate Plates Treated with Germlings of Ulva sp. J. World Aquac. Soc. 2012, 43, 890-895. [CrossRef]

51. Andras, T.D.; Alexander, T.S.; Gahlena, A.; Parry, R.M.; Fernandez, F.M.; Kubanek, J.; Wang, M.D.; Hay, M.E. Seaweed allelopathy against coral: Surface distribution of a seaweed secondary metabolite by imaging mass spectrometry. J. Chem. Ecol. 2012, 38, 1203-1214. [CrossRef]

52. Morrow, K.M.; Ritson-Williams, R.; Ross, C.; Liles, M.R.; Paul, V.J. Macroalgal extracts induce bacterial assemblage shifts and sublethal tissue stress in Caribbean corals. PLoS ONE 2012, 7, e44859. [CrossRef]

53. Rybak, A.S. Species of Ulva (Ulvophyceae, Chlorophyta) as indicators of salinity. Ecol. Indic. 2018, 85, 253-261. [CrossRef]

54. Rybak, A.S. The Ulva flexuosa complex (Ulvaceae, Chlorophyta): An updated identification key with special reference to the freshwater and hyperhaline taxa. Phytotaxa 2018, 345, 83-103. [CrossRef]

55. Paul, V.J.; Kuffner, I.B.; Walters, L.J.; Ritson-Williams, R.; Beach, K.S.; Becerro, M.A. Chemically mediated interactions between macroalgae Dictyota spp. and multiple life-history stages of the coral Porites astreoides. Mar. Ecol. Prog. Ser. 2011, 426, 161-170. [CrossRef]

56. Olsen, K.; Ritson-Williams, R.; Paul, V.J.; Ross, C. Combined effects of macroalgal presence and elevated temperature on the early life-history stages of a common Caribbean coral. Mar. Ecol. Prog. Ser. 2014, 509, 181-191. [CrossRef]

57. Vieira, C.; Thomas, O.P.; Culioli, G.; Genta-Jouve, G.; Houlbreque, F.; Gaubert, J.; De Clerc, O.; Payri, C.E. Allelopathic interactions between the brown algal genus Lobophora (Dictyotales, Phaeophyceae) and scleractinian corals. Sci. Rep. 2016, 6, 18637. [CrossRef]

58. El Gamal, A.A. Biological importance of marine algae. Saudi. Pharm. J. 2010, 18, 1-25. [CrossRef]

59. Tocher, R.D.; Craigie, J.S. Enzymes of marine algae: II. Isolation and identification of 3-hydroxytyramine as the phenolase substrate in Monostroma fuscum. Can. J. Bot. 1966, 44, 605-608. [CrossRef]

60. Paul, V.J.; Fenical, W. Isolation of halimedtrial: Chemical defense adaptation in the calcareous reef-building alga Halimeda. Science 1983, 221, 747-749. [CrossRef]

61. Wium-Andersen, S.; Anthoni, U.; Christophersen, C.; Houen, G. Allelopathic effects on phytoplankton by substances isolated from aquatic macrophytes (Charales). Oikos 1982, 39, 187-190. [CrossRef]

62. Wium-Andersen, S.; Anthoni, U.; Houen, G. Elemental sulphur, a possible allelopathic compound from Ceratophyllum demersum. Phytochemistry 1983, 22, 2613. [CrossRef]

63. Anthoni, U.; Nielsen, P.H.; Smith-Hansen, L.; Wium-Andersen, S.; Christophersen, C. Charamin, a quaternary ammonium ion antibiotic from the green alga Chara globularis. J. Org. Chem. 1987, 52, 694-695. [CrossRef]

64. Korzeniowska, K.; Łęska, B.; Wieczorek, P.P. Isolation and determination of phenolic compounds from freshwater Cladophora glomerata. Algal. Res. 2020, 48, 101912. [CrossRef]

65. Williams, D.E.; Sturgeon, C.M.; Roberge, M.; Andersen, R.J. Nigricanosides A and B antimitotic glycolipids isolated from the green alga Avrainvillea nigricans collected in Dominica. J. Am. Chem. Soc. 2007, 129, 5822-5823. [CrossRef]

66. Colon, M.; Guevara, P.; Gerwick, W.H.; Ballantine, D. 50-Hydroxyisoavrainvilleol, a new diphenylmethane derivative from the tropical green alga Avrainvillea nigricans. J. Nat. Prod. 1987, 50, 368-374. [CrossRef]

67. Chen, I.L.; Gerwick, W.H.; Schatzman, R.; Laney, M. Isorawsonol and related IMO dehydrogenase inhibitors from the tropical alga Avrainvillea rawsoni. J. Nat. Prod. 1994, 57, 947-952. [CrossRef] 
68. Hamann, M.T.; Scheuer, P. J Kahalalide F: A bioactive depsipeptide from the sacoglossan mollusk Elysia rufescens and the green alga Bryopsis sp. J. Am. Chem. Soc. 1993, 115, 5825-5826. [CrossRef]

69. Dmitrenok, A.; Iwashita, T.; Nakajima, T.; Sakamoto, B.; Namikoshi, M.; Nagai, H. New cyclic desipeptides from the green alga species; application of a carboxypeptidase hydrolysis reaction to the structure determination. Tetrahedron 2006, 62, 1301-1308. [CrossRef]

70. Wang, H.; Li, Y.-L.; Shen, W.-Z.; Rui, W.; Ma, X.-J.; Cen, Y.-Z. Antiviral activity of a sulfoquinovosyldiacylglycerol (SQDG) compound isolated from the green alga Caulerpa racemosa. Bot. Marina. 2007, 50, 185-190. [CrossRef]

71. Aguilar-Santos, G. Caulerpin, a new red pigment from green algae of the genus Caulerpa. J. Chem. Soc. C 1970, 6, 842-843. [CrossRef]

72. Ali, M.S.; Saleem, M.; Yammdagni, R.; Ali, M.A. Steroid and antibacterial glycosides from marine green alga Codium iyengarii Borgesen. Nat. Prod. Lett. 2002, 16, 407-413. [CrossRef]

73. Wall, M.E.; Wani, M.C.; Manikumar, G.; Taylor, H.; Gaetano, K.; Hughes, T.J.; Gerwick, W.H.; McPhail, A.T.; McPhail, D.R. Plant antimutagenic agents, structure and antimutagenic properties of cymobarbatol and 4-isocymobarbatol, new symbols from green alga Cymopolia barbata. J. Nat. Prod. 1989, 52, 1092-1099. [CrossRef]

74. Koehn, F.E.; Gunasekera, S.P.; Niel, D.N.; Cross, S.S. Halitunal, an unusual diterpene Aldehyde from the marine alga Halimeda tuna. Tetrahedron Lett. 1991, 32, 169-172. [CrossRef]

75. Paul, V.J.; Fenical, W. Novel bioactive diterpenoid metabolites from tropical marine algae of the genus Halimeda. Tetrahedron 1984, 40, 3053-3062. [CrossRef]

76. Puglisi, M.P.; Tan, L.T.; Jensen, P.R.; Fenical, W. Capisterones A and B from the tropical green alga Penicillus capitatus: Unexpected anti-fungal defenses targeting the marine pathogen Lindra thallasiae. Tetrahedron 2004, 60, 7035-7039. [CrossRef]

77. Govindan, M.; Abbas, S.A.; Schmitz, E.I.; Lee, R.H.; Papkoff, I.S.; Slate, D.L. New cycloartanol sulfates from the alga Tydemania expeditionis: Inhibitor of the protein tyrosin kinase pp60. J. Nat. Prod. 1994, 57, 74-78. [CrossRef]

78. Takahashi, C.; Numata, A.; Yamada, T.; Minoura, K.; Enomoto, S.; Konishi, K.; Nakai, M.; Matsuda, C.; Nomoto, K. Penostatins, novel cytotoxic metabolites from a Penicillium species separated from a green alga. Tetrahedron Lett. 1997, 37, 655-658. [CrossRef]

79. Iwamoto, C.; Minoura, K.; Hagishita, S.; Nomoto, K.; Numata, A. Penostatins F-I novel cytotoxic metabolites from a Penicillium species from an Enteromorpha marine alga. J. Chem. Soc. Perkin Trans. 1997, 3, 449-456. [CrossRef]

80. Iwamoto, C.; Minoura, K.; Hagishita, S.; Oka, T.; Ohta, T.; Hagishita, S.; Numata, A. Absolute sterostructures of novel penostatins A-E from a Penicillium species from an Enteromorpha marine alga. Tetrahedron 1999, 55, 14353-14368. [CrossRef]

81. Numata, A.; Takahashi, C.; Ito, Y.; Minoura, K.; Yamada, T.; Matsuda, C.; Nomoto, K. Penochalasins, a novel class of cytotoxic cytochalasans from a Penicillium species separated from a marine alga: Structure determination and solution conformation. J. Chem. Soc. Perkin Trans. 1996, 1, 239-245. [CrossRef]

82. Iwamoto, C.; Yamada, T.; Ito, Y.; Minoura, K.; Numata, A. Cytotoxic cytochalasans from a Penicillium species separated from a marine alga. Tetrahedron 2001, 57, 2904-2997. [CrossRef]

83. Numata, A.; Takahashi, C.; Ito, Y.; Takeda, T.; Kawai, K.; Osami, Y.; Matsumura, E.; Imachi, M.; Ito, T.; Hasegawa, T. Communesins, cytotoxic metabolites of a fungus isolated from a marine alga. Tetrahedron Lett. 1996, 34, 2355-2358. [CrossRef]

84. Awad, N.E. Biologically active steroid from the green alga Ulva lactuca. Phytother. Res. 2000, 14, 641-643. [CrossRef]

85. Ohta, K. Antimicrobial compounds in the marine red alga Beckerella subcostatum. Agric. Biol. Chem. 1977, 41, 2105-2106. [CrossRef]

86. Kubanek, I.; Prusak, A.C.; Snell, T.W.; Giese, R.A.; Hardcastle, K.I.; Fairchild, C.R.; Aalbersberg, W.; Raventos-Suarez, C.; Hay, M.E. Antineoplastic diterpene-benzoate macrolides from the Fijian red alga Callophycus serratus. Org. Lett. 2005, 7, 261-264. [CrossRef] [PubMed]

87. Kubanek, J.; Prusak, A.C.; Snell, T.W.; Giese, R.A.; Fairchild, C.R.; Aalbersberg, W.; Hay, M.E. Bromophycolides C-I from the Fijian red alga Callophycus serratus. J. Nat. Prod. 2006, 69, 731-735. [CrossRef]

88. Lane, A.L.; Stout, E.P.; Hay, M.E.; Prusak, A.C.; Hardcastle, K.; Fairchild, C.R.; Franzblau, S.G.; Le Roch, K.; Prudhomme, J.; Aalbersberg, W.; et al. Callophycoic acids and callophycols from the Fijian red alga Callophycus serratus. J. Org. Chem. 2007, 72, 7343-7351. [CrossRef]

89. Maeda, M.; Kodama, T.; Tanaka, T.; Yoshizumi, H.; Takemoto, T.; Nomoto, K.; Fujita, T. Structures of isodomic acids A, B and C novel insecticidal amino acids from the red alga Chonria armata. Chem. Pharm. Bull. 1986, 34, 4892-4895. [CrossRef]

90. Davyt, D.; Entz, W.; Fernandez, R.; Mariezcurrena, R.; Mombru, A.W.; Saldana, I.; Dominguez, L.; Coil, J.; Manta, E. A new indol derivative from the red alga chondra atropurpurea isolation, structure determination, and anthelmintic activity. J. Nat. Prod. 1998, 61, 1560-1563. [CrossRef]

91. Fenical, W.; Sims, J.J. Cycloeudesmol, an antibiotic cyclopropane conatinnin sequiterpene from the marine alga, Chondria oppositiclada Dawson. Tetrahedron Lett. 1974, 13, 1137-1140. [CrossRef]

92. Biscoe, T.J.; Evans, R.H.; Headley, P.M.; Martin, M.; Watkins, J.C. Domic and quisqualic acids as potent amino acids excitants of frog and rat spinal neurons. Nature 1975, 255, 166-167. [CrossRef]

93. Ferkany, J.W.; Coyle, J.T. Kainic acid selectively stimulates the release of endogenous excitatory acidic amino acids. J. Pharmacol. Exp. Therapeut. 1983, 225, 399-406.

94. Sun, Y.; Xu, Y.; Liu, K.; Hua, H.; Zhu, H.; Pei, Y. Gracilarioside and gracilamides from the red alga Gracilaria asiatica. J. Nat. Prod. 2006, 69, 1488-1491. [CrossRef] [PubMed] 
95. Ohata, K.; Mizushina, Y.; Hirata, N.; Sugawara, F.; Mutsukage, A.; Yoshida, S.; Sakaguchi, K. Sulphoquinovosy-ldiacylglycerol, KM043 a new potent inhibitor of eukaryotic DNA polymerases and HIV-reverse transcriptase type from a marine red alga Gigartina tenella. Chem. Pharm. Bull. 1998, 46, 684-686. [CrossRef]

96. Awad, N.E. Bioactive brominated diterpenes from the marine red alga Jania Rubens (L.) Lamx. Phytother Res. 2004, 18, 275-279. [CrossRef]

97. Carter, G.T.; Rinehart, K.L., Jr.; Li, L.H.; Kuentzel, S.L. Brominated indoles from Laurencia brongniartii. Tetrahedron Lett. 1978, 19, 4479-4482. [CrossRef]

98. El Gamal, A.A.; Wang, W.-L.; Duh, C.-Y. Sulfur-containing polybromoindoles from the Formosan red alga Laurencia brongniartii. J. Nat. Prod. 2005, 68, 815-817. [CrossRef]

99. Sims, J.J.; Lin, G.H.Y.; Wing, R.M. Marine natural products, elatol, a halogenated sesquiterpene alcohol from the red alga Laurencia elata. Tetrahedron Lett. 1974, 39, 3487-3490. [CrossRef]

100. Suzuki, T.; Furusaki, A.; Matsumoto, T.; Kato, A.; Lmanaka, Y.; Kurosawa, E. Teurilene and thyrsiferyl 23 acetate, meso and remarkably cytotoxic compounds from the marine red alga Laurencia obtuse. Tetrahedron Lett. 1985, 26, 1329-1332. [CrossRef]

101. Kladi, M.; Xenaki, H.; Vagias, C.; Papazafiri, P.; Roussis, V. New cytotoxic sesquiterpenes from the red algae Laurencia obtusa and Laurencia microcladia. Tetrahedron 2006, 62, 182-189. [CrossRef]

102. IIopoulou, D.; Mihopoulos, N.; Vigias, C.; Papazafiri, P.; Roussis, V. Novel cytotoxic brominated diterpenes from the red alga Laurencia obtuse. J. Org. Chem. 2003, 68, 7667-7674. [CrossRef]

103. Gonzalez, A.G.; Darias, J.; Diaz, A.; Fournero, J.D.; Martin, J.D.; Perez, C. Evidence for the biogenesis of halogenated chamigrenes from the red alga Laurencia obtusa. Tetrahedron Lett. 1976, 17, 3051-3054. [CrossRef]

104. Gonzalez, A.G.; Delgado, M.J.; Martin, V.S.; Martinez-Ripoll, M.; Fayos, J. X-ray study of sesquiterpene constituents of the alga Laurencia obtusa leads to structure revision. Tetrahedron Lett. 1979, 29, 2717-2718. [CrossRef]

105. Topeu, G.; Aydogmus, Z.; Imre, S.; Goren, A.C.; Pezzuto, J.M.; Clement, J.A.; Kinghorn, D.G. Brominated sesquiterpenes from the red alga Laurencia obtusa. J. Nat. Prod. 2003, 66, 1505-1508.

106. Norte, M.; Fernandez, J.J.; Saouto, M.L.; Gavin, J.A.; Garcia-Gravalos, M.D. Thyrsenols A and B two unusual polyether squalene derivatives. Tetrahedron 1997, 53, 3173-3178. [CrossRef]

107. Pec, M.K.; Aguirre, A.; Moser-Their, K.; Fernandez, J.J.; Souto, M.L.; Dota, J.; Diaz-Gonzalez, F.; Villar, J. Induction of apoptosis in estrogen dependent and independent breast cancer cells by the marine terpenoid dehydrothyrsiferol. Biochem. Pharmacol. 2003, 65, 1451-1461. [CrossRef]

108. Fukuzawa, A.; Masamune, T. Laurepinnacin and isolaurepinnacin: New acetylenic cyclic ethers from the marine alga Laurencia pinnata Yamada. Tetrahedron Lett. 1982, 22, 4081-4084. [CrossRef]

109. Gonzalez, A.G.; Martin, J.D.; Martin, V.S.; Norte, M.; Perez, R. Biomimetic approach to the synthesis of rhodolaureol and rhodolauradiol. Tetrahedron Lett. 1982, 23, 2395-2398. [CrossRef]

110. Ishii, T.; Nagamine, T.; Nguyen, B.C.Q.; Tawata, S. Insecticidal and repellent activities of laurinterol from the Okinawan red alga Laurencia nidifica. Rec. Nat. Prod. 2017, 11,63-68.

111. Watanabe, K.; Umeda, K.; Miyakado, M. Isolation and identification of three insecticidal principles from the red alga Laurencia nipponica Yamada. Agric. Biol. Chem. 1989, 53, 2513-2515. [CrossRef]

112. El Sayed, K.A.; Dunbar, D.C.; Perry, T.L.; Wilkins, S.P.; Hamann, M.T. Marine natural products as prototype insecticidal agents. J. Agric. Food Chem. 1997, 45, 2735-2739. [CrossRef]

113. Moon-Moo, K.; Sang-Hoon, L.; Se-Kwon, K. Patent from PCT Int. Appl. WO 2009048195 A1 20090416, 2009. (Language: English, Database: CAPLUS).

114. Davyt, D.; Fernandez, R.; Suescun, L.; Mombru, A.W.; Saldaña, J.; Domiínguez, L.; Fujii, M.T.; Manta, E. Bisabolanes from the red alga Laurencia scoparia. J. Nat. Prod. 2006, 69, 1113-1116. [CrossRef] [PubMed]

115. Sun, J.; Shi, D.Y.; Li, S.; Wang, S.J.; Han, L.J.; Fan, X.; Yang, Y.C.; Shi, J.G. Chemical constituents of the red alga Laurencia tristicha. J. Asian Nat. Prod. Res. 2007, 9, 725-734. [CrossRef]

116. Sakemi, S.; Higa, T.; Jefford, C.W.; Bernardinelli, G. Venustatriol: A new antiviral triterpene tetracyclic ether from Laurencia venusta. Tetrahedron Lett. 1986, 27, 4287-4290. [CrossRef]

117. Takahashi, Y.; Daitoh, M.; Suzuki, M.; Abe, T.; Masuda, M. Halogenated metabolites from the new Okinawan red alga Laurencia yonaguniensis. J. Nat. Prod. 2002, 65, 395-398. [CrossRef]

118. Gross, H.; Goeger, D.E.; Hills, P.; Mooberry, S.L.; Ballantine, D.L.; Murray, T.F.; Valeriote, F.A.; Gerwick, W.H. Lophocladines, bioactive alkaloids from the red alga Lophocladia sp. J. Nat. Prod. 2006, 69, 640-644. [CrossRef]

119. Bernari, M.W.; Gerwick, W.H. Eicosanoids from the tropical red alga Murrayella periclados. Phytochemistry 1994, 36, 1233-1240. [CrossRef]

120. Lee, H.-S.; Lee, T.-H.; Lee, J.H.; Chae, C.-S.; Chung, S.-C.; Shin, D.-S.; Shin, J.; Oh, K.-B. Inhibition of the pathogenicity of Magnaporthe grisea by bromophenols, isocitrate lyase inhibitors, from the red alga Odonthalia corymbifera. J. Agric. Food Chem. 2007, 55, 6923-6928. [CrossRef] [PubMed]

121. Talpir, R.; Rudi, A.; Kashman, Y.; Loya, Y.; Hizi, A. Three new sesquiterpene hydroquinones from marine origin. Tetrahedron 1994, 50, 4179-4184. [CrossRef]

122. Knott, M.G.; Mkwanazi, H.; Arendse, C.E.; Hendricks, D.T.; Bolton, J.J.; Beukes, D.R. Plocoralides A-C, polyhalogenated monoterpenes from the marine alga Plocamium corallorhiza. Phytochemistry 2005, 66, 1108-1112. [CrossRef] [PubMed] 
123. Watanabe, K.; Miyakado, M.; Ohno, N.; Okada, A.; Yanagi, K.; Moriguchi, K.A. A polyhalogenated isecticidal monoterepene from the red alga Plocamium telfairiae. Phytochemistry 1988, 28, 77-78. [CrossRef]

124. Lopez, A.; Gerwick, H. Ptiollodene, a novel eicosanoid inhibitor of 5 lipoxygenase and Na+/K+ ATPase from the red marine alga Ptilota filicina. Tetrahedron Lett. 1988, 29, 1505-1506. [CrossRef]

125. Wang, W.; Okada, Y.; Shi, H.; Wang, Y.; Okuyama, T. Tasipeptins A and B: Structures and aldose reductase inhibitory effects of bromophenols from the red alga Symphyocladia latiuscula. J. Nat. Prod. 2005, 68, 620-622. [CrossRef]

126. Wiemer, D.E.; Idler, D.D.; Fenical, W. Vidalols A and B, new antiinflammatory bromophenols from the Caribbean marine red alga Vidalia obtusaloba. Experientia 1991, 47, 851-853. [CrossRef]

127. Fisch, K.M.; Bohm, V.; Wrightand, A.D.; Konig, G.M. Antioxidative meroterpenoids from the brown alga Cystoseira crinita. J. Nat. Prod. 2003, 66, 968-975. [CrossRef]

128. Ayyad, S.-E.N.; Abdel-Halim, O.B.; Shier, W.T.; Hoye, T.R. Cytotoxic hydroazulene diterpenes from the brown alga Cystoseira myrica. Z. Natuforsch. C. Biosci. 2003, 58, 33-38. [CrossRef]

129. Bennamara, A.; Abourrichi, A.; Berrada, M.; Charrouf, M.H.; Chaib, N.; Boudouma, M.; Garneau, X.F. Methoxybifurcarenone: An antifungal and antibacterial meroditerpenoid from the brown alga Cystoseira tamariscifolia. Phytochemistry 1999, 52, 37-40. [CrossRef]

130. Muller, D.G.; Clayton, M.N.; Gassmann, O.; Boland, W.; Marner, F.J.; Schottes, T.; Jaenicke, L. Cystophorene and hormosirene, sperm attractants in Australian brown algae. Naturwissenschatien 1985, 72, 97-99. [CrossRef]

131. Ochi, M.; Kotsuki, H.; Muraoka, K.; Tokoroyama, T. The structure of yahazunol, a new sesquiterpene-substituted hydroquinone from the brown seaweed Dictyopteris undulata Okamura. Bull. Chem. Soc. Jpn. 1979, 52, 629-630. [CrossRef]

132. Kurata, K.; Tanguchi, K.; Suzuki, M. Cyclozonarone, a sesquiterpene-substituted benzoquinone derivative from the brown alga Dictyopteris undulate. Phytochemistry 1996, 41, 749-752. [CrossRef]

133. Fenical, W.; Sims, J.J.; Squatrito, D.; Wing, R.M.; Radlick, P. Marine natural products, VII Zonarol and isozonarol, fungitoxic hydroquinones from the brown seaweeds Dictyopteris zonarioides. J. Org. Chem. 1973, 38, 2383-2386. [CrossRef] [PubMed]

134. Barbosa, J.P.; Teixeira, V.L.; Villca, R.; Pereira, R.C.; Abrantes, J.L.; da Paixao Frugulhetti, I.C.P. A dolabellane diterpene from the Brazilian brown alga Dictyota pfaffii. J. Biochem. Syst. Ecol. 2003, 31, 1451-1453. [CrossRef]

135. Tringali, C.; Prattellia, M.; Nicols, G. Structure and conformation of new diterpenes based on the dolabellane skeleton from Dictyota species. Tetrahedron 1984, 40, 703-799. [CrossRef]

136. Ham, Y.M.; Baik, J.S.; Hyun, J.W.; Lee, N.H. Isolation of a new phlorotannin, fucodiphlorethol G, from a brown alga Ecklonia cava. Bull. Korean Chem. Soc. 2007, 28, 1595-1597.

137. Kang, H.S.; Chang, H.Y.; Jung, J.H.; Son, B.W.; Choi, J.S. A new phlorotannin from the brown alga Ecklonia stolonifera. Chem. Pharm. Bull. 2003, 51, 1012-1014. [CrossRef]

138. Boland, W.; Jaenicke, L.; Muller, D.G.; Gassmann, G. Giffordene, 2Z, 4Z, 6E, 8Z-undecatetraene, is the odoriferous principle of the marine brown alga Giffordia mitchellae. Experientia 1987, 43, 466-468. [CrossRef]

139. Edmonds, S.1.; Morita, M.; Shibata, Y. Isolation and identification of Arsenic containing ribfurnaoside and inorganic Arsenic from Japanes edible seaweed Hizikia fusiforme. J. Chem. Soc. Perkin. Trans. I 1987, 577-580. [CrossRef]

140. Yamada, T.; Iwamoto, C.; Yamagaki, N.; Yamanouchi, T.; Minoura, K.; Yamori, T.; Uehara, Y.; Andoh, T.; Umemura, K.; Numata, A. Leptosins M-N Cytotoxic metabolites from a Leptosphaeria species separated from a marine alga, structure determination and biological activities. Tetrahedron 2002, 58, 479-487. [CrossRef]

141. Kubanek, J.; Jensen, P.R.; Keifer, P.A.; Sullards, M.C.; Collins, D.O.; Fenical, W. Seaweed resistance to microbial attack: A targeted chemical defense against marine fungi. Proc. Nat. Acad. Sci. USA 2003, 100, 6916-6921. [CrossRef]

142. Capon, R.I.; Barrow, R.A.; Rochfort, S.; Jobling, M.; Skene, C.; Larcey, E.; Gill, I.H.; Friedel, T.; Wadsworth, D. Marine Nematodes: Tetrahydrofuran from a southern Australian brown alga, Notheia anomala. Tetrahdron 1998, 54, 2227-2242. [CrossRef]

143. Barreto, M.; Meyer, J.J.M. Isolation and antimicrobial activity of a lanosol derivative from Osmundaria serrata (Rhodophyta) and a visual exploration of its biofilm covering. S. Afr. J. Bot. 2006, 72, 521-528. [CrossRef]

144. Muller, D.G.; Boland, W.; Becker, U.; Wahl, T. Caudoxirene, the spermatozide-releasing and attracting factor in the marine brown alga Perithalia caudate (Phaeophyceae, Sporochnales). Biol. Chem. Hopper-Seyler 1988, 369, 655-659. [CrossRef] [PubMed]

145. Lee, Y.S.; Shin, K.H.; Kim, B.K.; Lee, S. Anti-diabetic activities of fucosterol from Pelvetia siliquosa. Arch. Pharmacal. Res. 2004, 27, 1120-1122. [CrossRef]

146. Jang, K.H.; Lee, H.B.; Choi, B.W.; Lee, H.-S.; Shin, J. Chromenes from the brown alga Sargassum siliquastrum. J. Nat. Prod. 2005, 68, 716-723. [CrossRef]

147. Numata, A.; Kambara, S.; Takahashi, C.; Fujiki, R.; Yoneda, M.; Fujita, E.; Nabeshima, Y. Cytotoxic activity of marine algae and a cytotoxic principle of the brown alga Sargassum tortile. Chem. Pharm. Bull. 1991, 39, 2129-2131. [CrossRef]

148. Numata, A.; Kanbara, S.; Takahashi, C.; Fujiki, R.; Yoneda, M.; Usami, Y.; Fujita, E. A cytotoxic principle of the brown alga Sargassum tortile and structures of chromenes. Phytochemistry 1992, 31, 1209-1213. [CrossRef]

149. Seo, Y.; Park, K.E.; Kim, Y.A.; Lee, H.-I.; Yoo, I.-S.; Ahn, I.-W.; Lee, B.-J. Isolation of tetraprenyltoluquinols from the brown alga Sargassum thunbergii. Chem. Pharm. Bull. 2006, 54, 1730-1733. [CrossRef] [PubMed]

150. Seo, Y.; Park, K.E.; Nam Bull, T.J. Isolation of a new chromene from the brown alga Sargassum thunbergii. Korean Chem. Soc. 2007, $28,1831-1833$ 
151. Kim, Y.H.; Kim, E.-H.; Lee, C.; Kim, M.-H.; Rho, J.-R. Two new monogalactosyl diacylglycerols from brown alga Sargassum thunbergii. Lipids 2007, 42, 395-399. [PubMed]

152. Rovirosa, J.; Sepulveda, M.; Quezada, E.; San-Martin, A. Isoepitaondiol, a diterpenoid of Stypopodium flabelliforme and the insecticidal activity of stypotriol Epitaondiol and derivatives. Phytochemistry 1992, 31, 2679-2681. [CrossRef]

153. Dorta, E.; Cueto, M.; Diaz-Marrero, A.R.; Darias, J. Stypolactone an interesting diterpenoid from the brown alga Stypopodium zonale. Tetrahedron Lett. 2002, 65, 9043-9046. [CrossRef]

154. Wessels, M.; Konig, G.M.; Wright, A.D. A new tyrosine kinase inhibitor from the marine brown alga Stypopodium zonale. J. Nat. Prod. 1999, 62, 927-930. [CrossRef]

155. Othmani, A.; Bunet, R.; Bonnefont, J.L.; Briand, J.F.; Culioli, G. Settlement inhibition of marine biofilm bacteria and barnacle larvae by compounds isolated from the Mediterranean brown alga Taonia atomaria. J. Appl. Physiol. 2016, 28, 1975-1986. [CrossRef]

156. Tziveleka, L.-A.; Abatis, D.; Paulus, K.; Bauer, R.; Vigias, C.; Roussis, V. Marine polyprenylated hydroquinones, quinones, and chromenols with inhibitory effects on leukotriene formation. Chem. Biol. 2005, 2, 901-909. [CrossRef]

157. Abatis, D.; Vigias, C.; Galanakis, D.; Norris, J.N.; Moreau, D.; Roussakis, C.; Rousis, V. Atomarianones A and B: Two cytotoxic meroditerpenes from the brown alga Taonia atomaria. Tetrahedron Lett. 2005, 46, 8525-8529. [CrossRef]

158. Asari, F.; Kusumi, T.; Kakisawa, H. Turbinaric acid, a cytotoxic secosqualene carboxylic acid from the brown alga Turbinaria ornate. J. Nat. Prod. 1989, 52, 1167-1169. [CrossRef] [PubMed]

159. Céspedes, C.L.; Avila, J.G.; Martínez, A.; Serrato, B.; Calderón-Mugica, J.C.; Salgado-Garciglia, R. Antifungal and antibacterial activities of Mexican tarragon (Tagetes lucida). J. Agric. Food Chem. 2006, 54, 3521-3527. [CrossRef]

160. Li, X.; Wang, J.; Huang, D.; Wang, L.; Wang, K. Allelopathic potential of Artemisia frigida and successional changes of plant communities in the northern China steppe. Plant Soil 2011, 341, 383-398. [CrossRef]

161. Talukdar, D. Allelopathic effects of Lantana camara L. on Lathyrus sativus L.: Oxidative imbalance and cytogenetic consequences. Allelopath. J. 2013, 31, 71-90.

162. Yu, Z.W.; Sun, W.H.; Guo, K.Q. Allelopathic effects of several aquatic plants on algae. Acta Hydrobiol. Sin. 1992, 16, 1-7.

163. Shannon-Firestone, S.; Firestone, J. Allelopathic potential of invasive species is determined by plant and soil community context. Plant Ecol. 2015, 216, 491-502. [CrossRef]

164. Abrahim, D.; Braguini, W.L.; Kelmer-Bracht, A.M.; Ishii- Iwamoto, E.L. Effects of four monoterpenes on germination, primary root growth, and mitochondrial respiration of maize. J. Chem. Ecol. 2000, 26, 611-624. [CrossRef]

165. Śliwińska-Wilczewska, S.; Budzałek, G.; Kowalska, Z.; Klin, M.; Latała, A. Baltic macroalgae as a potential source for commercial applications-review. Ann. Univ. Paedagog. Crac. Studia Nat. 2020, 5, 220-237.

166. Lubchenco, J.; Gaines, S.D. A unified approach to marine plant-herbivore interactions: I. Populations and communities. Annu. Rev. Ecol. Evol. Syst. 1981, 12, 405-437. [CrossRef]

167. Hay, M.E.; Fenical, W. Marine plant-herbivore interactions: The ecology of chemical defense. Annu. Rev. Ecol. Evol. Syst. 1988, 19, 111-145. [CrossRef]

168. Reigosa, M.J.; Sánchez-Moreiras, A.; González, L. Ecophysiological approach in allelopathy. Crit. Rev. Plant Sci. 1999, 18, 577-608. [CrossRef]

169. Chao, L.; Levin, B.R. Structured habitats and the evolution of anticompetitor toxins in bacteria. Proc. Natl. Acad. Sci. USA 1981, 78, 6324-6328. [CrossRef]

170. Antonelli, P.L.; Sammarco, P.W.; Coll, J.C. A model of allelochemical interactions between soft and scleractinian corals on the Great Barrier Reef. J. Biol. Syst. 1993, 1, 1-17. [CrossRef]

171. Cheng, H.H. Characterization of the mechanisms of allelopathy-modeling and experimental approaches. ACS Sym. Ser. 1995, 582, 132-141.

172. Tapaswi, P.K.; Mukhopadhyay, A. Effects of environmental fluctuation on plankton allelopathy. J. Math. Biol. 1999, 39, 39-58. [CrossRef]

173. An, M.; Johnson, I.R.; Lovett, J.V. Mathematical modelling of residue allelopathy the effects of intrinsic and extrinsic factors. Plant Soil 2002, 246, 11-22. [CrossRef]

174. An, M.; Liu, D.L.; Johnson, I.R.; Lovett, J.V. Mathematical modelling of allelopathy. II. The dynamics of allelochemicals from living plants in the environment. Ecol. Model. 2003, 161, 53-66. [CrossRef] 